\title{
Teoria de Grupos e o Papel das Simetrias em Física
}

\author{
Group theory and the role of symmetries in physics
}

\author{
J. Furtado*1@, J. A. Helaÿel-Neto ${ }^{2}$ \\ ${ }^{1}$ Universidade Federal do Cariri, Centro de Ciências e Tecnologia, Juazeiro do Norte, CE, Brasil. \\ ${ }^{2}$ Centro Brasileiro de Pesquisas Físicas, Rio de Janeiro, RJ, Brasil.
}

\begin{abstract}
Recebido em 12 de agosto de 2020. Revisado em 28 de outubro de 2020. Aceito em 03 de novembro de 2020.
Este artigo tem como objetivo principal apresentar, de maneira sucinta e intuitiva, aspectos fundamentais da teoria de grupos, estabelecendo sua relação com a ideia de simetria, traçando um paralelo com o desenvolvimento da mecânica quântica e da física de partículas. Buscamos abordar este tema, que é frequentemente negligenciado em cursos de graduação, partindo sempre do conceito de invariância de uma dada quantidade física de interesse para, só então, introduzir a ideia de grupo de simetria.
\end{abstract}

Palavras-chave: Teoria de grupos, invariância, física de partículas.

\begin{abstract}
This paper sets out to present, in a brief and intuitive way, fundamental aspects of group theory, establishing its relation with the idea of symmetry, drawing a parallel with the development of quantum mechanics and particle physics. We try to approach this topic, which is frequently neglected in undergraduate courses, always starting from the concept of invariance of a given quantity of interest to, only after that, introduce the idea of a symmetry group.
\end{abstract}

Keywords: Group theory, invariance and symmetry, particle physics.

\section{Introdução}

No início do Século XX, a revolução científica promovida pelo desenvolvimento da Mecânica Quântica e da Teoria da Relatividade permitiu que déssemos mais um passo na busca pela resposta à seguinte pergunta: de quê são feitas as coisas? A Mecânica Quântica restringiu o domínio de validade da Física Newtoniana para corpos macroscópicos, enquanto que a Teoria da Relatividade Restrita impôs à Mecânica Clássica o domínio de baixas velocidades. Na tentativa de compreender o elétron e a sua estrutura interna caracterizada pelo spin, Dirac propõe uma teoria quântica relativística para o elétron [1].

Até o ano de 1931 acreditava-se que o universo fosse constituído por apenas três partículas, a saber, o próton, o elétron e o fóton. O elétron foi descoberto por J.J. Thomson em 1897 ao realizar experimentos com raios catódicos 2]. O próton, cujas primeiras evidências foram vistas por Eugene Goldstein em seus experimentos com raios canais [3], foi efetivamente descoberto enquanto partícula por Ernest Rutherford em seu famoso experimento do espalhamento de partículas $\alpha$ (átomos de hélio ionizados) em uma fina folha de ouro [4. Já o fóton, que teve suas primeiras evidências detectadas em 1900, foi tema de intenso debate [5]. Devido à ausência de massa, a comunidade científica da época permaneceu relutante por muito tempo em relação à

\footnotetext{
*Endereço de correspondência: job.furtado@ufca.edu.br
}

atribuição do caráter de partícula ao fóton. Somente após os experimentos de Compton em 1923 foi que o fóton recebeu o status de partícula [6].

Em 1930, o seminal trabalho intitulado The Proton [7] é publicado por Dirac. Neste trabalho, Dirac discutia acerca da possibilidade de o próton ser a antipartícula do elétron, só que em estado excitado, uma vez que a massa do próton é cerca de 1800 vezes a massa do elétron. No ano seguinte, influenciado por Hermann Weyl [8] e suas ideias sobre teoria de grupos, Dirac descarta a possibilidade de o próton ser a antipartícula do elétron e publica um trabalho que discorre acerca da possível antipartícula do elétron, da quantização da carga elétrica e dos monopólos magnéticos 9 .

No ano de 1932, a descoberta da antipartícula do elétron (o pósitron), um gêmeo positivamente carregado do elétron, com todos os atributos que Dirac havia previsto, consolida a teoria relativística do elétron e marca uma nova era na física de partículas [10. Apesar da influência de certa forma indireta da teoria de grupos no desenvolvimento da teoria relativística do elétron, a busca por simetrias na natureza levou, naturalmente, a comunidade científica da época a olhar mais atentamente para as estruturas de grupos, uma vez que estas estabelecem uma generalização natural da ideia de simetria.

Após o ano de 1932, uma série de novas partículas e propriedades foi sendo descobertas. A descrição matemática natural de tais partículas e propriedades, sempre visando a ideia de unificação e simetria, foi através da teoria de grupos. O "caminho do octeto", proposto 
por Gell-Mann em 1961, arranja mésons (partículas compostas por dois quarks) e bárions (partículas compostas por três quarks) em padrões geométricos regulares, organizando e classificando a física de partículas [11]. O trabalho de Gell-Mann foi realizado com base nos resultados fenomenológicos e experimentais que se tinha. Fundamentado matematicamente em teoria de grupos, mais especificamente no grupo SU(3), o trabalho de GellMann marca praticamente o início da teoria de grupos como ferramenta base no estudo da física de partículas. Desde então, a teoria de grupos vem sendo cada vez mais utilizada na descrição, fundamentação e previsão de física nova, tendo se tornado um atributo essencial na descrição coerente de sistemas físicos, em especial em física de altas energias.

O conceito de grupo formaliza a ideia de simetria, isto quer dizer que, num sentido amplo, a ideia de simetria pode ser entendida através de invariantes por grupos de transformações. Neste trabalho focaremos no estudo dos grupos matriciais contínuos conhecidos como grupos de Lie. De modo geral, um grupo de Lie é a combinação de uma estrutura algébrica de grupo com a estrutura de uma variedade diferenciável. Os grupos que hoje conhecemos como grupos de Lie foram inicialmente estudados por Sophus Lie por volta de 1870, buscando compreender equações diferenciais a partir de seus grupos de simetria [12]. Os métodos para se estudar grupos de Lie estão baseados em suas álgebras associadas, isto é, nas álgebras de Lie. Uma vez munido da álgebra de Lie associada a um grupo de Lie qualquer, é possível obter propriedades globais ou locais do grupo a partir da álgebra. A vantagem de se adotar esta abordagem (que será a abordagem adotada neste trabalho) consiste no fato de que, sendo os grupos objetos tipicamente não lineares, estudar a álgebra de Lie associada tornase bem mais simples, uma vez que a álgebra é linear, o que permite que sejam utilizados resultados clássicos e poderosos da álgebra linear.

Este trabalho está organizado como se segue. Na seção 2 abordamos de maneira intuitiva a ideia de grupo, partindo do conceito de invariância, e usando como base o grupo $\mathrm{SO}(2)$. Encontramos os geradores do grupo e sua álgebra, conceitos que serão trabalhados em todas as seções subsequentes, e descrevemos a relação entre o grupo $\mathrm{SO}(2)$ e as rotações no plano. $\mathrm{Na}$ seção 3 trabalhamos com o grupo U(1), discutindo sua relação com o grupo $\mathrm{SO}(2)$ e o eletromagnetismo. $\mathrm{Na}$ seção 4 discutimos o grupo $\operatorname{Sp}(2)$, detalhando sua álgebra e estabelecendo a conexão com o espaço AdS. Na seção 5 abordamos o grupo SU(2) partindo da ideia de invariância do produto interno no espaço complexo, conectando esta ideia ao conceito de conservação de probabilidade em mecânica quântica. A seção 6 consistirá da exposição da relação existente entre o grupo de rotações em três dimensões, o grupo $\mathrm{SO}(3)$, e o grupo $\mathrm{SU}(2)$. Abordamos ainda aspectos algébricos do grupo SU(3). E por fim discutimos o oscilador harmônico unidimensional, bidimensional e N-dimensional, a fim de explicitar sua correspondência com os grupos unitários.

\section{Noção Intuitiva e Grupo $\mathrm{SO}(2)$}

Suponha que estejamos interessados em encontrar uma transformação que deixe a energia de um sistema, como por exemplo, o oscilador harmônico, inalterada. Podemos descrever a energia de um oscilador harmônico através da seguinte equação:

$$
E=\frac{p^{2}}{2 m}+\frac{1}{2} k x^{2} .
$$

Desta forma, buscar uma transformação que não altere a energia de tal sistema consiste em tentar encontrar uma transformação $R$ que não mude o estado de energia do sistema, isto é, $E^{\prime}=E$. Sabemos ainda que a quantidade $p^{2}$ é formalmente descrita através do produto escalar entre $\vec{p}$ e $\vec{p}$, isto é, $p^{2}=\vec{p} \cdot \vec{p}$, bem como $x^{2}=\vec{x} \cdot \vec{x}$.

Aqui o conceito fundamental é a ideia de invariância. Note ainda que uma das formas de efetuar uma transformação a fim de manter $E$ invariante é encontrar uma transformação $R$ que mantenha o produto escalar em si invariante. Buscamos então encontrar uma transformação que atue em vetores de modo a não alterar o produto escalar, isto é, uma transformação $R$ tal que sendo $\vec{v}^{\prime}=R \vec{v}$ tenhamos $\vec{v}^{\prime} \cdot \vec{v}^{\prime}=\vec{v} \cdot \vec{v}$.

Para tal vamos relembrar que o produto interno entre dois vetores $\vec{v}=\left(v_{1}, v_{2}\right)$ e $\vec{w}=\left(w_{1}, w_{2}\right)$ é definido como

$$
\vec{v} \cdot \vec{w}=v_{1} w_{1}+v_{2} w_{2} .
$$

Aqui consideramos que $\vec{v}, \vec{w} \in \mathbb{R}^{2}$, muito embora a definição acima seja válida para um espaço euclidiano com dimensão arbitrária. Os dois vetores $\vec{v}$ e $\vec{w}$ acima descritos podem ser expressos matricialmente como

$$
V=\left(\begin{array}{l}
v_{1} \\
v_{2}
\end{array}\right), \quad W=\left(\begin{array}{l}
w_{1} \\
w_{2}
\end{array}\right) .
$$

Usando a notação matricial, o produto interno entre esses dois vetores pode ser escrito da seguinte maneira

$$
\begin{aligned}
V^{T} W & =\left(\begin{array}{ll}
v_{1} & v_{2}
\end{array}\right)\left(\begin{array}{l}
w_{1} \\
w_{2}
\end{array}\right) \\
& =v_{1} w_{1}+v_{2} w_{2}=\vec{v} \cdot \vec{w} .
\end{aligned}
$$

Partindo então da notação matricial, estamos interessados em uma transformação $R$ tal que o produto interno permaneça invariante. Note que, sendo $\vec{v}$ e $\vec{w}$ pertencentes a $\mathbb{R}^{2}$, então a matriz de transformação $R$ deve pertencer ao espaço das matrizes de ordem 2 com entradas reais, que denotaremos por $\mathbb{M}(2, \mathbb{R})$. Definindo os vetores $V^{\prime}=R V$ e $W^{\prime}=R W$, o produto escalar entre $V^{\prime}$ e $W^{\prime}$ passa a ser escrito como:

$$
V^{\prime T} W^{\prime}=V^{T} R^{T} R W .
$$

Vemos então que, para que o produto escalar seja invariante, é necessário que $R^{T} R=I$, sendo $I$ a matriz 
identidade de ordem 2. Note que, dessa forma, temos que $R^{T}=R^{-1}$. Matrizes cujas transpostas são iguais às suas inversas são chamadas de matrizes ortogonais.

Vamos então definir o conjunto de todas as matrizes ortogonais de ordem 2 com entradas reais. Tal conjunto é escrito como:

$$
O(2)=\left\{M \in \mathbb{M}(2, \mathbb{R}) ; M^{T}=M^{-1}\right\} .
$$

Sobre o conjunto $\mathrm{O}(2)$ acima descrito podemos ver que $I \in \mathrm{O}(2)$, uma vez que a $I=I^{T}=I^{-1}$. Além disso, dadas duas matrizes $M_{1}, M_{2} \in \mathrm{O}(2)$, o produto $M_{1} M_{2} \in$ $\mathrm{O}(2)$, pois,

$$
\left(M_{1} M_{2}\right)^{T}=M_{2}^{T} M_{1}^{T}=M_{2}^{-1} M_{1}^{-1}=\left(M_{1} M_{2}\right)^{-1} .
$$

Outra propriedade que pode ser notada é que, dada uma matriz $M \in \mathrm{O}(2)$, a matriz $M^{-1} \in \mathrm{O}(2)$. Tal propriedade é consequência do fato de $\left(M^{-1}\right)^{T}=$ $\left(M^{T}\right)^{-1}$. E por fim, a última propriedade que pode ser imediatamente verificada é que, dadas três matrizes $M_{1}, M_{2}, M_{3} \in \mathrm{O}(2)$, então é valido que:

$$
M_{1}\left(M_{2} M_{3}\right)=\left(M_{1} M_{2}\right) M_{3} .
$$

Dizemos então que um dado conjunto não vazio $\mathrm{G}$, munido de uma operação $(\cdot)$ é chamado de grupo se são satisfeitas as quatro propriedades acima listadas para o conjunto (que de agora em diante chamaremos de grupo) $\mathrm{O}(2)$, isto é:

$$
\begin{gathered}
g_{1} \cdot g_{2} \in G \forall g_{1}, g_{2} \in G, \\
g_{1} \cdot\left(g_{2} \cdot g_{3}\right)=\left(g_{1} \cdot g_{2}\right) \cdot g_{3} \forall g_{1}, g_{2}, g_{3} \in G, \\
\exists e \in G ; g \cdot e=e \cdot g=g \forall g \in G, \\
\exists g^{-1} \in G ; g^{-1} \cdot g=g \cdot g^{-1}=e \forall g \in G .
\end{gathered}
$$

Nas equações acima a quantidade e é chamada de elemento neutro do grupo, justamente por possuir a propriedade de que quando multiplicada (aqui entenda multiplicação como uma operação arbitrária a ser definida) por qualquer elemento $g$ do grupo resulta no próprio elemento $g$.

Agora que definimos formalmente a estrutura de um grupo, vamos analisar mais atentamente as matrizes pertencentes ao grupo $\mathrm{O}(2)$. Uma característica importante do grupo $\mathrm{O}(2)$ (na verdade sendo válida para o grupo $\mathrm{O}(\mathrm{n})$, que é uma generalização do grupo $\mathrm{O}(2)$ para matrizes com qualquer ordem $n$ ) é que as matrizes a ele pertencentes têm determinante $\operatorname{det} M= \pm 1$.

Demonstração: Considerando uma matriz $M \in \mathrm{O}(2)$, $\log O$

$$
\begin{aligned}
1 & =\operatorname{det}(\hat{I}) \\
& =\operatorname{det}\left(M^{-1} M\right)=\operatorname{det}\left(M^{T} M\right) \\
& =\operatorname{det}\left(M^{T}\right) \operatorname{det}(M)=\operatorname{det}(M)^{2} \\
& \Rightarrow \operatorname{det}(M)= \pm 1,
\end{aligned}
$$

como queríamos demonstrar. Desta forma, fica claro que o grupo $\mathrm{O}(2)$ contém duas categorias de subconjuntos, um subconjunto formado por matrizes com $\operatorname{det}(M)=+1$ e outro por matrizes $\operatorname{com} \operatorname{det}(M)=-1$, de modo que definimos,

$$
\begin{aligned}
X & =\{M \in O(2) ; \operatorname{det}(M)=-1\} \\
\mathrm{SO}(2) & =\{M \in O(2) ; \operatorname{det}(M)=+1\} .
\end{aligned}
$$

Note que o conjunto $X$ nem mesmo é grupo, uma vez que $I \notin X$. Vamos agora estudar mais detalhadamente o grupo $\mathrm{SO}(2) \subset \mathrm{O}(2)$. Considere uma matriz $M \in \mathrm{SO}(2)$ arbitrária,

$$
M=\left(\begin{array}{ll}
a & b \\
c & d
\end{array}\right) .
$$

De acordo com a definição do grupo $\mathrm{SO}(2), M^{T}=M^{-1}$, o que implica em

$$
\left(\begin{array}{ll}
a & c \\
b & d
\end{array}\right)=\left(\begin{array}{cc}
d & -b \\
-c & a
\end{array}\right)
$$

Logo, $a=d$ e $b=-c$. Como $\operatorname{det} M=1$, obtemos a seguinte relação $a^{2}+b^{2}=1$ entre os elementos de $M$. Isto nos diz que $(a, b)$ deve estar no círculo unitário. Agora veja que $\forall M \in \mathrm{SO}(2)$, temos:

$$
M=\left(\begin{array}{cc}
a & b \\
-b & a
\end{array}\right)
$$

com $a^{2}+b^{2}=1$. A condição expressa sobre $a$ e $b$ nos permite tomar a seguinte transformação:

$$
\begin{aligned}
a & =\cos \theta \\
b & =\operatorname{sen} \theta
\end{aligned}
$$

onde $\theta \in(-\pi, \pi), \log \mathrm{O}$

$$
M=M(\theta)=\left(\begin{array}{cc}
\cos \theta & \operatorname{sen} \theta \\
-\operatorname{sen} \theta & \cos \theta
\end{array}\right) .
$$

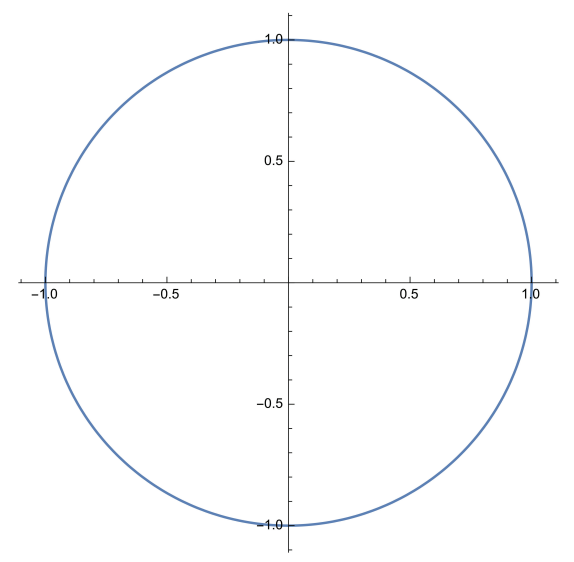

Figura 1: Círculo unitário. 
Portanto,

$$
\mathrm{SO}(2)=\left\{M(\theta)=\left(\begin{array}{cc}
\cos \theta & \operatorname{sen} \theta \\
-\operatorname{sen} \theta & \cos \theta
\end{array}\right) ; \theta \in(-\pi, \pi)\right\}
$$

onde $M(0)=\hat{I}$. Considere agora um vetor $\vec{v}=(1,0)$, que pode ser expresso como um vetor coluna $V$,

$$
V=\left(\begin{array}{l}
1 \\
0
\end{array}\right)
$$

e uma matriz $M\left(90^{\circ}\right)$ que é escrita como:

$$
M=M\left(90^{\circ}\right)=\left(\begin{array}{cc}
0 & 1 \\
-1 & 0
\end{array}\right) .
$$

A ação de $M\left(90^{\circ}\right)$ em $V$, isto é, o vetor $V^{\prime}=M\left(90^{\circ}\right) V$ é dado por:

$$
V^{\prime}=\left(\begin{array}{c}
0 \\
-1
\end{array}\right)
$$

Geometricamente, isso pode ser visto na Figura 2. Portanto, a ação de $M \in \mathrm{SO}(2)$ em um dado vetor promove a rotação deste vetor sobre um ângulo $\theta$. A ação de $M \in \mathrm{SO}(2)$ também pode ser interpretada como uma rotação passiva do sistema de coordenadas no sentido anti-horário, como pode ser visto na Figura 3. Outra propriedade que pode ser verificada de maneira imediata é que, dados dois ângulos $\theta_{1}$ e $\theta_{2}$, as matrizes de transformação associadas a tais ângulos obedecem a seguinte relação:

$M\left(\theta_{1}\right) M\left(\theta_{2}\right)=M\left(\theta_{1}+\theta_{2}\right)=M\left(\theta_{2}+\theta_{1}\right)=M\left(\theta_{2}\right) M\left(\theta_{1}\right)$

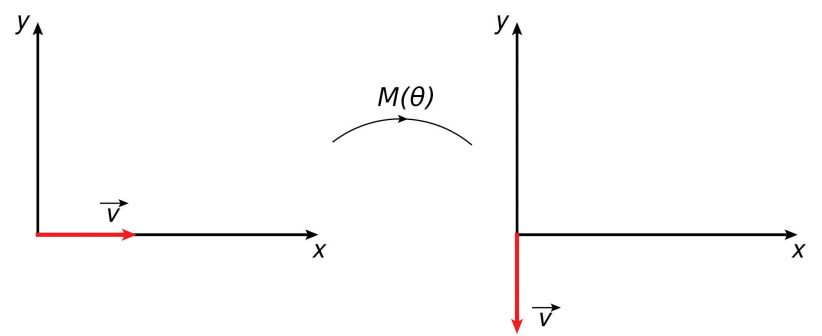

Figura 2: Ação de $M(\theta)$.

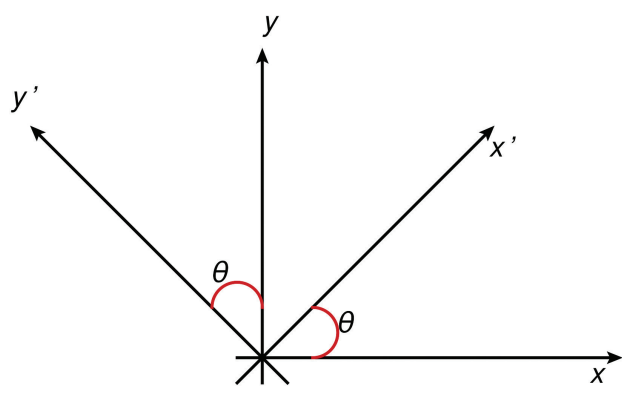

Figura 3: Rotação. o que mostra que o grupo $\mathrm{SO}(2)$ é um grupo comutativo (ou abeliano).

É importante ressaltar aqui que, como vimos anteriormente, o grupo $\mathrm{SO}(2)$ surge como uma consequência da imposição de uma invariância no produto escalar. Estando o produto escalar entre dois vetores relacionados a módulos e ângulos, é natural que a matriz de rotação seja uma possível representação do grupo $\mathrm{SO}(2)$ (que é também conhecido como grupo de rotações em duas dimensões), uma vez que rotacionar dois vetores por um mesmo ângulo preserva o produto escalar entre eles.

Analogamente ao que foi feito no caso do grupo $\mathrm{SO}(2)$, podemos parametrizar os elementos das matrizes $R \in X$ em termos de funções trigonométricas, de modo que,

$$
R=R(\theta)=\left(\begin{array}{cc}
\cos \theta & \operatorname{sen} \theta \\
\operatorname{sen} \theta & -\cos \theta
\end{array}\right)
$$

Note ainda que a identidade não se encontra no conjunto, explicitando que este não possui a estrutura de grupo. Além do mais, qualquer elemento do grupo pode ainda ser escrito como

$$
R(\theta)=\left(\begin{array}{cc}
1 & 0 \\
0 & -1
\end{array}\right) M(\theta)
$$

Portanto, concluímos que toda matriz $R \in X$ pode ser escrita como apresentada na equação anterior, o que representa uma rotação rígida e seguida de uma reflexão.

Voltando à análise do grupo $\mathrm{SO}(2)$, é sabido que qualquer matriz que possui determinante igual à unidade pode ser escrita como a exponencial [13] de uma matriz que possui traço nulo. De modo que existe $\Omega \in \mathbb{M}(2, \mathbb{R})$ tal que

$$
M=e^{\Omega}
$$

sendo $\operatorname{Tr}(\Omega)=0$. Portanto, como $M \in \mathrm{SO}(2)$, temos que,

$$
M^{T}=M^{-1} \Rightarrow \Omega^{T}=-\Omega .
$$

Desta forma, tomando uma matriz arbitrária $\Omega$

$$
\Omega=\left(\begin{array}{ll}
a & b \\
c & d
\end{array}\right)
$$

e impondo a condição acima, obtemos

$$
\Omega=b \varepsilon=b\left(\begin{array}{cc}
0 & 1 \\
-1 & 0
\end{array}\right)
$$

A matriz $\varepsilon$, conhecida como matriz simplética, é o que chamamos de gerador do grupo, enquanto que $b$ é chamado de parâmetro do grupo. Note ainda que, se tomarmos $b=\theta$, a matriz $M$ passa a ser escrita como

$$
M=e^{\theta \varepsilon} .
$$


Veja que $\varepsilon^{2}=-I$. Agora vamos expandir em série a quantidade $e^{\theta \varepsilon}$

$$
\begin{aligned}
e^{\theta \varepsilon} & =\sum_{k=0}^{\infty} \frac{1}{k !} \theta^{k} \varepsilon^{k} \\
& =\sum_{l=0}^{\infty} \frac{1}{(2 l) !} \theta^{2 l} \varepsilon^{2 l}+\sum_{l=0}^{\infty} \frac{1}{(2 l+1) !} \theta^{2 l+1} \varepsilon^{2 l+1}
\end{aligned}
$$

$\mathrm{Na}$ última passagem, separamos a soma em seus termos pares e ímpares. Vamos agora usar as seguintes identidades,

$$
\begin{aligned}
\varepsilon^{2 l} & =(-1)^{l} I \\
\varepsilon^{2 l+1} & =(-1)^{l} \varepsilon,
\end{aligned}
$$

portanto,

$$
\begin{aligned}
e^{\theta \varepsilon} & =\sum_{l=0}^{\infty} \frac{1}{(2 l) !} \theta^{2 l}(-1)^{l} I+\sum_{l=0}^{\infty} \frac{1}{(2 l+1) !} \theta^{2 l+1}(-1)^{l} \varepsilon \\
& =\cos \theta I+\sin \theta \varepsilon \\
& =\left(\begin{array}{cc}
\cos \theta & \operatorname{sen} \theta \\
-\operatorname{sen} \theta & \cos \theta
\end{array}\right)=M(\theta)
\end{aligned}
$$

Vemos assim que todo elemento do grupo pode ser escrito como a exponenciação de seus geradores. O grupo $\mathrm{SO}(2)$ é um grupo de dimensão 1, uma vez que o grupo é inteiramente determinado pela matriz simplética. A ideia por trás do conceito de geradores de um grupo está na capacidade de expressar todos os elementos de um dado grupo através da exponenciação seus geradores. O leitor pode ainda perceber que a matriz simplética é a representação matricial do tensor Levi-Civita $\left(\varepsilon_{i j}, i, j=\right.$ $\{1,2\})$ de ordem 2. O tensor de Levi-Civita é tal que $\varepsilon_{i j}=0$ se $i=j, \varepsilon_{12}=+1$ e $\varepsilon_{21}=-1$.

Quando o elemento geral de um grupo depende apenas de um parâmetro $(\theta$, no caso do grupo $\mathrm{SO}(2))$, dizemos que tal grupo é um grupo uniparamétrico. Para tais grupos, o gerador pode ser calculado através da seguinte relação:

$$
\varepsilon=\left.\frac{d M(\theta)}{d \theta}\right|_{\theta=0} .
$$

Os conceitos desenvolvidos durante esta seção serão utilizados para o desenvolvimento das seções que se seguem.

\section{Grupo U(1) e a Eletrodinâmica}

Até este ponto ficou claro que dado um vetor $\vec{v} \in \mathbb{R}^{2}$, que pode ser escrito em forma de vetor como $\vec{v}=(x, y)$ ou em forma de matriz

$$
V=\left(\begin{array}{l}
x \\
y
\end{array}\right)
$$

escrito em um sistema de coordenadas $S$, pode ser relacionado a um vetor $\vec{v}^{\prime} \in \mathbb{R}^{2}$ de coordenadas $\vec{v}^{\prime}=\left(x^{\prime}, y^{\prime}\right)$ em um sistema de coordenadas $S^{\prime}$ que foi passivamente rotacionado em relação ao sistema $S$ de um ângulo $\theta$, como na Figura 3

A relação entre tais vetores é tal que

$$
\left(\begin{array}{l}
x^{\prime} \\
y^{\prime}
\end{array}\right)=R(\theta)\left(\begin{array}{l}
x \\
y
\end{array}\right),
$$

sendo $R(\theta)$ uma matriz pertencente ao grupo $\mathrm{SO}(2)$, portanto:

$$
\left(\begin{array}{l}
x^{\prime} \\
y^{\prime}
\end{array}\right)=\left(\begin{array}{cc}
\cos \theta & \operatorname{sen} \theta \\
-\operatorname{sen} \theta & \cos \theta
\end{array}\right)\left(\begin{array}{l}
x \\
y
\end{array}\right) .
$$

Agora considere $z \in \mathbb{C}$ escrito como $z=x+i y$ e uma matriz $W$ escrita como:

$$
W=\left(\begin{array}{c}
z \\
z^{*}
\end{array}\right) .
$$

Qual grupo está por trás da conexão entre $W$ e $W^{\prime}$ ?

$$
\begin{aligned}
& \left(\begin{array}{l}
x^{\prime} \\
y^{\prime}
\end{array}\right) \stackrel{M(\theta)}{\longrightarrow}\left(\begin{array}{l}
x \\
y
\end{array}\right), \\
& \left(\begin{array}{c}
z^{\prime} \\
z^{\prime *}
\end{array}\right) \stackrel{?}{\longrightarrow}\left(\begin{array}{c}
z \\
z^{*}
\end{array}\right) .
\end{aligned}
$$

Para tal investigação lembremos inicialmente que em $\mathbb{R}^{2}$, a conexão é tal que $V^{\prime}=M(\theta) V$, o que nos dá que

$$
\begin{aligned}
& x^{\prime}=\cos \theta x-\operatorname{sen} \theta y \\
& y^{\prime}=\operatorname{sen} \theta x+\cos \theta y .
\end{aligned}
$$

A partir da definição de $z$ como um número complexo da forma $z=x+i y$, podemos escrever $x$ e $y$ como sendo

$$
\begin{aligned}
& x=\frac{z+z^{*}}{2} \\
& y=\frac{z-z^{*}}{2 i},
\end{aligned}
$$

o que consequentemente nos permite escrever $x^{\prime}$ e $y^{\prime}$, fazendo uso das equações (43) e (44), em termos de $z$ e $z^{*}$ da seguinte forma

$$
\begin{aligned}
x^{\prime} & =\frac{z}{2} e^{i \theta}+\frac{z^{*}}{2} e^{-i \theta} \\
i y^{\prime} & =\frac{z}{2} e^{i \theta}-\frac{z^{*}}{2} e^{-i \theta} .
\end{aligned}
$$

Portanto, escrevendo naturalmente $z^{\prime}=x^{\prime}+i y^{\prime}$ bem como $z^{* \prime}=x^{\prime}-i y^{\prime}$ chegamos às seguintes leis de transformação

$$
\begin{aligned}
z^{\prime} & =e^{i \theta} z \\
z^{* \prime} & =e^{-i \theta} z^{*} .
\end{aligned}
$$

Note portanto que, diferentemente da lei de transformação entre $V$ e $V^{\prime}$, em que as componentes $x^{\prime}$ e $y^{\prime}$ dependem simultaneamente de $x$ e $y$, no caso da relação entre $W$ e $W^{\prime}$ as componentes desacoplam, isto é, $z^{\prime}$ 
transforma só com $z$ (eq. (49)) e $z^{*^{\prime}}$ transforma só com $z^{*}$ (eq. 50). Podemos então escrever

$$
\left(\begin{array}{c}
z^{\prime} \\
z^{*^{\prime}}
\end{array}\right)=\left(\begin{array}{cc}
e^{i \theta} & 0 \\
0 & e^{-i \theta}
\end{array}\right)\left(\begin{array}{c}
z \\
z^{*}
\end{array}\right) .
$$

Como as equações desacoplam, podemos usar somente a eq. 49 para explicitar a relação entre $z^{\prime}$ e $z$, que é feita através de uma matriz pertencente à $M(\mathbb{C}, 1)$, isto é $e^{i \theta}$ tal que a sua inversa é igual à sua transposta conjugada. Este conjunto define um grupo conhecido como $U(1)$, explicitamente definido como:

$$
U(1)=\left\{Z \in M(\mathbb{C}, 1) ; Z^{-1}=Z^{\dagger}\right\}
$$

Tal grupo preserva o módulo de $z$ perante transformação, porém não sua fase angular, sendo a relação 49 muitas vezes conhecida como transformação de fase. Vemos assim que, o grupo U(1) possui dimensão 1, possui um único gerador e que os elementos do grupo são matrizes de ordem 1, isto é, um número. Em teoria de grupos dizemos que, quando o número de geradores de um grupo for igual à ordem das matrizes do grupo, estaremos diante de uma representação adjunta ou regular daquele grupo. Discutiremos a importância da representação adjunta dos grupos mais adiante.

Um ponto importante que deve ser ressaltado aqui é a íntima relação do grupo U(1) com a eletrodinâmica. Mesmo que o leitor não esteja familiarizado com a ação para férmions relativísticos, o presente exemplo se faz instrutivo. Considere a seguinte ação

$$
S=\int d^{4} x \bar{\psi}\left(i \gamma^{\mu} \partial_{\mu}-m\right) \psi
$$

que descreve férmions relativísticos. Sendo $\gamma^{\mu}$ com $\mu=\{0,1,2,3\}$ as matrizes de Dirac e $m$ a massa do férmion. A ação acima é invariante perante a seguinte transformação:

$$
\psi \longrightarrow e^{i \theta} \psi
$$

Veja então que a quantidade $e^{i \theta} \in \mathrm{U}(1)$. Se considerarmos uma fase local, o que implica em impor uma dependência em $\theta$, isto é, tomar $\theta=\theta(x)$, a invariância é perdida, uma vez que,

$$
\begin{aligned}
\bar{\psi}^{\prime} i \gamma^{\mu} \partial_{\mu} \psi^{\prime} & =\bar{\psi} i \gamma^{\mu} \partial_{\mu} \psi-\bar{\psi} \gamma^{\mu}\left(\partial_{\mu} \theta\right) \psi \\
& \neq \bar{\psi} i \gamma^{\mu} \partial_{\mu} \psi
\end{aligned}
$$

Para recuperar a invariância sob transformações de U(1) com uma fase local é necessário definir uma derivada, conhecida como derivada covariante, da seguinte maneira:

$$
D_{\mu}=\partial_{\mu}-i e A_{\mu}
$$

e a seguinte lei de transformação para $A_{\mu} \rightarrow A_{\mu}^{\prime}=$ $A_{\mu}+(1 / e)\left(\partial_{\mu} \theta\right)$, sendo $A_{\mu}=\left(A_{0}, A_{i}\right)$ o campo de gauge, com $A_{0}$ sendo o potencial escalar e $A_{i}$ as componentes do potencial vetor. Desta forma, identificando a quantidade e com a carga elétrica, o grupo $\mathrm{U}(1)$ torna-se o "responsável" pela interação entre os férmions com o campo eletromagnético (representado pelo campo de gauge $A_{\mu}$ ). E assim começa a aparecer a estrutura matemática por trás da QED (quantum electrodynamics), descrita essencialmente pela lagrangiana:

$$
\mathcal{L}=-\frac{1}{4} F_{\mu \nu} F^{\mu \nu}+\bar{\psi}\left(i D_{\mu} \gamma^{\mu}-m\right) \psi
$$

$\operatorname{com} F_{\mu \nu}=\partial_{\mu} A_{\nu}-\partial_{\nu} A_{\mu}$

\section{Grupo $\operatorname{Sp}(2)$ e o Espaço $\operatorname{AdS}_{3}$}

Já vimos que o grupo $\mathrm{SO}(2)$ mantém invariante o produto interno entre dois vetores quaisquer e que, portanto, preserva comprimentos e ângulos. Considere agora dois vetores no plano $\vec{v}=\left(v_{1}, v_{2}\right)$ e $\vec{w}=\left(w_{1}, w_{2}\right)$. O produto vetorial em duas dimensões também pode ser escrito em forma matricial como

$$
\begin{aligned}
Z=V^{T} \varepsilon W & =\left(\begin{array}{ll}
v_{1} & v_{2}
\end{array}\right)\left(\begin{array}{cc}
0 & 1 \\
-1 & 0
\end{array}\right)\left(\begin{array}{l}
w_{1} \\
w_{2}
\end{array}\right) \\
& =v_{1} w_{2}-v_{2} w_{1}=\vec{v} \times \vec{w} .
\end{aligned}
$$

Veja que a matriz simplética, além de ser o gerador do grupo $\mathrm{SO}(2)$, é utilizada também para estabelecer a representação matricial do produto vetorial em duas dimensões. Considere agora uma transformação de $\mathrm{SO}(2)$ atuando em dois vetores $V$ e $W$. De modo que definamos $V^{\prime}=M(\theta) V$ e $W^{\prime}=M(\theta) W$. Desta forma, o produto vetorial entre $V^{\prime}$ e $W^{\prime}$ passa a ser escrito como:

$$
\begin{aligned}
Z^{\prime} & =\left(V^{\prime}\right)^{T} \varepsilon W^{\prime} \\
& =[M(\theta) V]^{T} \varepsilon M(\theta) W \\
& =V^{T} M(\theta)^{T} \varepsilon M(\theta) W .
\end{aligned}
$$

É fácil ver que $M(\theta)^{T} \varepsilon M(\theta)=\varepsilon$ e que portanto uma transformação de $\mathrm{SO}(2)$ deixa $Z$ invariante, isto é, $Z^{\prime}=Z$. Uma questão natural que surge é a seguinte: o grupo de transformações de $\mathrm{SO}(2)$ é o conjunto mais geral de transformações que deixa o produto vetorial invariante? A fim de responder tal questão podemos escrever uma matriz $S \in M(2, \mathbb{R})$ arbitrária tal que:

$$
S^{T} \varepsilon S=\varepsilon .
$$

Sendo $S$ definida como:

$$
S=\left(\begin{array}{ll}
a & b \\
c & d
\end{array}\right)
$$

a relação 60 nos dá que:

$$
\left(\begin{array}{cc}
0 & a d-b c \\
-a d+b c & 0
\end{array}\right)=\left(\begin{array}{cc}
0 & 1 \\
-1 & 0
\end{array}\right) .
$$

Da relação acima obtemos o seguinte vínculo:

$$
a d-b c=1 \rightarrow \operatorname{det}(S)=1 .
$$


Portanto, a transformação mais geral que deixa o produto vetorial invariante requer uma matriz cujo determinante seja igual à unidade. Note então que,

$$
\mathrm{SO}(2) \Rightarrow\left\{\begin{array}{l}
a=d=\cos \theta \\
b=-c=\operatorname{sen} \theta
\end{array}\right.
$$

o grupo $\mathrm{SO}(2)$ é um caso particular de uma matriz $M$ que deixa o produto vetorial invariante. Assim, podemos definir um conjunto

$$
\operatorname{Sp}(2)=\left\{S \in M(2, \mathbb{R}) ; S^{T} \varepsilon S=\varepsilon\right\}
$$

como o conjunto das matrizes $S$ que deixa invariante o produto vetorial. É possível então mostrar que $\operatorname{Sp}(2)$ é grupo. Tal grupo é conhecido como grupo simplético em duas dimensões. O vínculo imposto nas matrizes de $\operatorname{Sp}(2)$ que implica que seus determinantes sejam iguais à unidade (eq. 63p) estabelece que na matriz de $\operatorname{Sp}(2)$ há três parâmetros livres e que, portanto, o grupo $\mathrm{Sp}(2)$ tem dimensão igual a três. Assim como no caso do grupo $\mathrm{SO}(2)$, ao qual está associado o espaço geométrico do círculo unitário, pode-se perguntar qual a geometria associada ao grupo $\operatorname{Sp}(2)$. Note que a relação 63 é uma quádrica, e que portanto pode ser expressa como:

$$
\begin{aligned}
a d-b c & =\frac{1}{2}\left(\begin{array}{llll}
a & b & c & d
\end{array}\right)\left(\begin{array}{cccc}
0 & 0 & 0 & 1 \\
0 & 0 & -1 & 0 \\
0 & -1 & 0 & 0 \\
1 & 0 & 0 & 0
\end{array}\right)\left(\begin{array}{l}
a \\
b \\
c \\
d
\end{array}\right) \\
& =\frac{1}{2}\left(\begin{array}{llll}
a & b & c & d
\end{array}\right) T\left(\begin{array}{l}
a \\
b \\
c \\
d
\end{array}\right)
\end{aligned}
$$

A matriz $T$ acima é simétrica e possui autovalores $\tau= \pm 1$, sendo portanto diagonalizável. Desta forma, pelo teorema espectral, existe uma matrix $J$ ortogonal [13] tal que:

$$
J T J^{T}=\left(\begin{array}{cccc}
1 & 0 & 0 & 0 \\
0 & 1 & 0 & 0 \\
0 & 0 & -1 & 0 \\
0 & 0 & 0 & -1
\end{array}\right) .
$$

Desta forma podemos inserir dois operadores identidade na forma $J^{T} J$ de modo que a quantidade $a d-b c$ assume a seguinte forma:

$$
a d-b c=\tilde{a}^{2}+\tilde{b}^{2}-\tilde{c}^{2}-\tilde{d}^{2}=1 .
$$

Note, portanto, que a equação anterior define um hiperboloide (ver Figura 4). Isso significa dizer que os elementos do grupo $\mathrm{Sp}(2)$ vivem sob a superfície de um hiperboloide. Tal geometria é também conhecida como $A d S_{3}$.

O espaço $A d S_{3}$ (Anti de Sitter em 3-dimensões) é um espaço muito estudado em física, tanto no que diz respeito à física de altas energias, como em física da

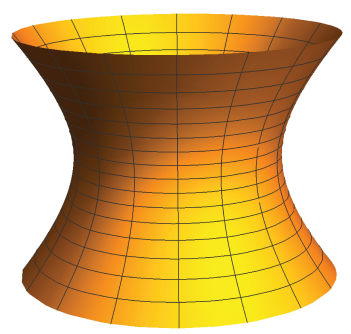

Figura 4: Hipersuperfície $(\tilde{d}=0)$ do hiperboloide em 4 dimensões.

matéria condensada. Em um trabalho futuro pretendemos discutir com mais detalhes a íntima relação entre as estruturas de grupo dos espaços do tipo de Sitter, anti de Sitter e as dualidades em física de altas energias.

Vamos agora discutir os geradores e a álgebra associada ao grupo $\mathrm{Sp}(2)$. Como já é sabido que qualquer matriz de determinante igual à unidade pode ser escrita como a exponencial de uma matriz de traço zero, estamos procurando uma matriz $\Omega$ tal que

$$
M=e^{\Omega} .
$$

Desta forma, a relação 60 nos dá que

$$
\left(e^{\Omega}\right)^{T} \varepsilon e^{\Omega}=\varepsilon \Rightarrow e^{\Omega^{T}} \varepsilon e^{\Omega}=\varepsilon .
$$

A fim de encontrar $\Omega$, podemos expandir as exponenciais até primeira ordem, de modo que

$$
\left[1+\Omega^{T}\right] \varepsilon[1+\Omega]=\varepsilon .
$$

Distributindo e excluindo o termo de segunda ordem, obtemos a seguinte relação

$$
\varepsilon \Omega=-\Omega^{T} \varepsilon \Rightarrow \varepsilon \Omega \varepsilon=\Omega^{T} .
$$

O motivo pelo qual consideramos apenas termos de primeira ordem no desenvolvimento acima ficará mais claro adiante. Se definirmos uma matriz $\Omega$ arbitrária e usarmos a relação 72 obtemos uma matriz $\Omega$ tal que $\operatorname{tr}(\Omega)=0$, como esperado, com três parâmetros livres, o que nos diz que $\operatorname{dim}[\operatorname{Sp}(2)]=3$. De modo geral $\Omega$ pode ser escrito da seguinte forma

$$
\begin{aligned}
\Omega & =\alpha\left(\begin{array}{cc}
1 & 0 \\
0 & -1
\end{array}\right)+\beta\left(\begin{array}{ll}
0 & 1 \\
0 & 0
\end{array}\right)+\gamma\left(\begin{array}{ll}
0 & 0 \\
1 & 0
\end{array}\right) \\
& =\alpha \lambda_{1}+\beta \lambda_{2}+\gamma \lambda_{3} .
\end{aligned}
$$

As matrizes $\lambda_{i}$ definem os geradores do grupo $\operatorname{Sp}(2)$, cuja álgebra de Lie associada (álgebra $\mathbf{s p}(2)$ ) é expressa por suas relações de comutação, isto é, dadas duas matrizes $A$ e $B$, definimos o comutador entre $A$ e $B$ como $[A, B]=$ $A B-B A$. Portanto, a álgebra $\mathbf{s p}(2)$ é expressa por:

$$
\begin{aligned}
& {\left[\lambda_{1}, \lambda_{2}\right]=2 \lambda_{2},} \\
& {\left[\lambda_{1}, \lambda_{3}\right]=-2 \lambda_{3},} \\
& {\left[\lambda_{2}, \lambda_{3}\right]=\lambda_{1} .}
\end{aligned}
$$


De maneira heurística, pode-se dizer que, para grupos de Lie [12], a seguinte relação é sempre válida:

$$
G=e^{A},
$$

sendo $G$ o grupo e $A$ a álgebra associada ao grupo. Note que as matrizes que pertencem ao grupo não obrigatoriamente pertencem à álgebra, por exemplo, a matriz identidade pertence ao grupo $\mathrm{Sp}(2)$, mas não à álgebra $\mathbf{s p}(2)$, visto que não possui traço nulo.

\section{Grupo SU(2) e a Mecânica Quântica}

Na interpretação de Copenhague da mecânica quântica [14] a função de onda $\psi(\vec{r}, t)$ tem o papel fundamental de indicar a amplitude de probabilidade de que uma dada partícula encontre-se em uma caixa infinitesimal $d V=$ $d x d y d z$, centrada em $\vec{r}=(x, y, z)$, em um dado instante de tempo $t$. De modo que a densidade de probabilidade é dada por:

$$
|\psi(\vec{r}, t)|^{2}=\langle\psi, \psi\rangle=\psi^{*} \psi
$$

Na notação de Dirac, por exemplo, o estado quântico de um sistema é dado por um vetor que pertence ao espaço dos estados, digamos, $|\psi\rangle \in \mathbb{E}$. Enquanto que a amplitude de probabilidade de que este estado arbitrário $|\psi\rangle$ encontre-se no estado $|\alpha\rangle$ é dada pelo produto interno entre estes dois vetores, contudo, como tais vetores de modo geral estão em $\mathbb{C}^{n}$, o produto interno obedece uma forma sesquilinear. Para o presente objetivo vamos considerar dois vetores $z=\left(z_{1}, z_{2}\right)$ e $w=\left(w_{1}, w_{2}\right)$ em $\mathbb{C}^{2}$. O produto interno entre estes dois vetores é dado por:

$$
\langle z \mid w\rangle=z_{1}^{*} w_{1}+z_{2}^{*} w_{2}
$$

Assim como foi feito anteriormente, podemos expressar este produto interno em forma matricial, bastando, para isso, definir

$$
Z=\left(\begin{array}{l}
z_{1} \\
z_{2}
\end{array}\right), \quad W=\left(\begin{array}{l}
w_{1} \\
w_{2}
\end{array}\right)
$$

e assim, é possível notar que

$$
\begin{aligned}
Z^{\dagger} W & =\left(\begin{array}{ll}
z_{1}^{*} & z_{2}^{*}
\end{array}\right)\left(\begin{array}{l}
w_{1} \\
w_{2}
\end{array}\right) \\
& =z_{1}^{*} w_{1}+z_{2}^{*} w_{2}=\langle z \mid w\rangle
\end{aligned}
$$

É importante lembrar aqui que $Z^{\dagger}=\left(Z^{T}\right)^{*}$. Portanto, a pergunta imediata que surge é saber se existe uma transformação que deixe o produto interno em $\mathbb{C}$ invariante. Assim como no caso do grupo $\mathrm{SO}(2)$, a grande importância em encontrar uma transformação tal que mantenha o produto interno em $\mathbb{C}$ invariante reside no fato de que desta forma encontraremos transformações que preservarão a probabilidade de um sistema quântico.
Analogamente, consideraremos vetores transformados $Z^{\prime}=Q Z$ e $W^{\prime}=Q W$, então

$$
Z^{\prime \dagger} W^{\prime}=Z^{\dagger} Q^{\dagger} Q W=\langle Q Z \mid Q W\rangle
$$

Para manter invariância é necessário, desta forma, que $Q^{\dagger} Q=I$ o que implica em $Q^{\dagger}=Q^{-1}$. Matrizes com a propriedade tal que o transposto conjugado seja igual à inversa são chamadas de matrizes unitárias. É possível mostrar que o conjunto

$$
U(2)=\left\{Q \in \mathbb{M}(2, \mathbb{C}) ; U^{\dagger}=U^{-1}\right\}
$$

forma um grupo. Tal grupo é conhecido como grupo unitário em duas dimensões. Vamos investigar agora algumas das propriedades básicas de matrizes $Q \in \mathrm{U}(2)$. Considere uma matriz $Q \in \mathrm{U}(2)$ arbitrária

$$
Q=\left(\begin{array}{ll}
a & b \\
c & d
\end{array}\right)
$$

A condição $Q^{\dagger}=Q^{-1}$ nos dá o seguinte vínculo entre os elementos da matriz

$$
\left(\begin{array}{ll}
a^{*} & c^{*} \\
b^{*} & d^{*}
\end{array}\right)=\frac{1}{\operatorname{det}(Q)}\left(\begin{array}{cc}
d & -b \\
-c & a
\end{array}\right) .
$$

Da equação anterior obtemos as seguintes equações

$$
\left\{\begin{array}{l}
d=\operatorname{det}(Q) a^{*} \\
a=\operatorname{det}(Q) d^{*}
\end{array}\right.
$$

Quando combinamos as equações acima obtemos que $|\operatorname{det}(Q)|=1$. Portanto, toda e qualquer matriz $Q \in \mathrm{U}(2)$ possui determinante com módulo igual a unidade. Vamos nos deter ao subconjunto $\mathrm{SU}(2) \subset \mathrm{U}(2)$ das matrizes que possuem $\operatorname{det}(Q)=1$, isto é,

$$
\mathrm{SU}(2)=\{Q \in U(2) ; \operatorname{det}(Q)=1\} .
$$

Impondo a condição de $\mathrm{SU}(2)$ na matriz $Q$ vemos que a forma geral de uma matriz de $\mathrm{SU}(2)$ é

$$
Q=\left(\begin{array}{cc}
a & b \\
-b^{*} & a^{*}
\end{array}\right)
$$

de modo que a condição $\operatorname{det}(Q)=1$ implica em $|a|^{2}+|b|^{2}=1$. Buscando agora investigar em qual espaço geométrico os elementos do grupo $\mathrm{SU}(2)$ vivem, é conveniente escrever a matriz $Q$ em termos de suas componentes reais, de modo que

$$
Q=\left(\begin{array}{cc}
\alpha+i \beta & \gamma+i \delta \\
-\gamma+i \delta & \alpha-i \beta
\end{array}\right)
$$

e assim, a condição imposta pelo determinante nos diz que

$$
\alpha^{2}+\beta^{2}+\gamma^{2}+\delta^{2}=1
$$

determinando assim uma esfera $S_{3}$. 


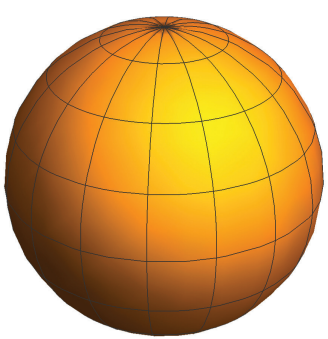

Figura 5: Hipersuperfície $(\delta=0)$ da esfera em 4 dimensões.

Veja que a matriz (90) pode ser expandida em uma base, da seguinte maneira

$$
\begin{aligned}
\left(\begin{array}{cc}
\alpha+i \beta & \gamma+i \delta \\
-\gamma+i \delta & \alpha-i \beta
\end{array}\right)= & \alpha\left(\begin{array}{ll}
1 & 0 \\
0 & 1
\end{array}\right)+\beta\left(\begin{array}{cc}
i & 0 \\
0 & -i
\end{array}\right) \\
& +\gamma\left(\begin{array}{cc}
0 & 1 \\
-1 & 0
\end{array}\right)+\delta\left(\begin{array}{ll}
0 & i \\
i & 0
\end{array}\right) \\
= & \alpha I+i \delta \sigma_{1}+i \gamma \sigma_{2}+i \beta \sigma_{3} .
\end{aligned}
$$

sendo as matrizes $\sigma_{1}, \sigma_{2}$ e $\sigma_{3}$ chamadas de matrizes de Pauli e definidas como

$$
\begin{gathered}
\sigma_{1}=\left(\begin{array}{ll}
0 & 1 \\
1 & 0
\end{array}\right) \\
\sigma_{2}=\left(\begin{array}{cc}
0 & -i \\
i & 0
\end{array}\right) \\
\sigma_{3}=\left(\begin{array}{cc}
1 & 0 \\
0 & -1
\end{array}\right) .
\end{gathered}
$$

Portanto, o conjunto composto pela matriz identidade e pelas matrizes $\left\{i \sigma_{1}, i \sigma_{2}, i \sigma_{3}\right\}$ formam uma base para o grupo $\mathrm{SU}(2)$. Além disso, o conjunto formado pelas matrizes $\left\{i \sigma_{1}, i \sigma_{2}, i \sigma_{3}\right\}$ define o sistema numérico conhecido como números quaterniônicos (representados pela letra $\mathbb{H}$ ), que é uma extensão dos números complexos. Maiores detalhes sobre os quatérnions podem ser vistos em [15]. A fim de encontrar os geradores do grupo $\mathrm{SU}(2)$, a matriz $\Omega$ cuja exponenciação dará origem aos elementos do grupo $\mathrm{SU}(2)$ deverá ser tal que

$$
\left(e^{\Omega}\right)^{\dagger}=\left(e^{\Omega}\right)^{-1} \Rightarrow \Omega^{\dagger}=-\Omega .
$$

Como uma consequência da imposição acima e do fato de que a matriz $\Omega$ possui traço nulo, esta passa a ser escrita genericamente como:

$$
\Omega=\left(\begin{array}{cc}
a & b \\
-b^{*} & -a
\end{array}\right)
$$

com a sendo um número puramente imaginário. De modo que podemos expressar a matriz $\Omega$ da seguinte forma

$$
\begin{aligned}
\Omega & =\left(\begin{array}{cc}
i \alpha & \beta+i \gamma \\
-\beta+i \gamma & -i \alpha
\end{array}\right)=i \gamma \sigma_{1}+i \beta \sigma_{2}+i \alpha \sigma_{3} \\
& =i \Delta
\end{aligned}
$$

com $\Delta=\gamma \sigma_{1}+\beta \sigma_{2}+\alpha \sigma_{3}$. Assim, podemos expressar a matriz $Q$ em termos de $\Delta$ como $Q=e^{i \Delta}$, e a condição imposta em $\Delta$ é tal que

$$
\left(e^{\Omega}\right)^{\dagger}=\left(e^{\Omega}\right)^{-1} \Rightarrow \Delta^{\dagger}=\Delta .
$$

Dizemos então que as matrizes de Pauli $\left\{\sigma_{1}, \sigma_{2}, \sigma_{3}\right\}$ são os geradores do grupo $\mathrm{SU}(2)$. Antes de desenvolver mais detalhadamente a álgebra $\mathbf{s u}(\mathbf{2})$, é importante notar que a matriz identidade não pertence à álgebra su(2), uma vez que tais matrizes devem ser hermitianas e ter traço nulo. A matriz identidade é hermitiana, porém não possui traço nulo.

A álgebra de Lie $\mathbf{s u ( 2 )}$ é dada pelas relações de comutação entre os geradores do grupo associado, isto é, o grupo $\mathrm{SU}(2)$. A partir do grupo $\mathrm{SU}(2)$ podemos obter também uma álgebra de Clifford (para mais detalhes vide [16]), dada pelas relações de anticomutaçã ${ }^{1}$ entre os geradores do grupo associado. Um cálculo direto nos mostra que tais relações são as seguintes:

$$
\begin{aligned}
{\left[\sigma_{1}, \sigma_{2}\right] } & =\sigma_{1} \sigma_{2}-\sigma_{2} \sigma_{1}=2 i \sigma_{3}, \\
{\left[\sigma_{2}, \sigma_{3}\right] } & =\sigma_{2} \sigma_{3}-\sigma_{3} \sigma_{2}=2 i \sigma_{1}, \\
{\left[\sigma_{3}, \sigma_{1}\right] } & =\sigma_{3} \sigma_{1}-\sigma_{1} \sigma_{3}=2 i \sigma_{2}, \\
\left\{\sigma_{1}, \sigma_{1}\right\} & =\left\{\sigma_{2}, \sigma_{2}\right\}=\left\{\sigma_{3}, \sigma_{3}\right\}=2 I, \\
\left\{\sigma_{1}, \sigma_{2}\right\} & =\left\{\sigma_{2}, \sigma_{3}\right\}=\left\{\sigma_{3}, \sigma_{1}\right\}=0 .
\end{aligned}
$$

As relações de comutação e anticomutação podem ser simplificadas através do uso da notação tensorial

$$
\begin{aligned}
{\left[\sigma_{a}, \sigma_{b}\right] } & =\sigma_{a} \sigma_{b}-\sigma_{b} \sigma_{a}=2 i \sum_{c=1}^{3} \varepsilon_{a b c} \sigma_{c} \\
\left\{\sigma_{a}, \sigma_{b}\right\} & =\sigma_{a} \sigma_{b}+\sigma_{b} \sigma_{a}=2 \delta_{a b} \hat{I}
\end{aligned}
$$

e a seguinte identidade pode ser facilmente verificada

$$
\sigma_{a} \sigma_{b}=\delta_{a b} \hat{I}+i \sum_{c=1}^{3} \varepsilon_{a b c} \sigma_{c}
$$

Nas equações acima os índices livres podem assumir valores $\{1,2,3\}$, o delta de Kronecker $\delta_{a b}=0$ se $a \neq b$ e $\delta_{a b}=1$ se $a=b$. O tensor de Levi-Civita em três dimensões é tal que $\varepsilon_{a b c}=+1$ se $\{a, b, c\}$ for qualquer permutação par de $\{1,2,3\}, \varepsilon_{a b c}=-1$ se $\{a, b, c\}$ for qualquer permutação ímpar de $\{1,2,3\}$ e zero se houver índices repetidos, isto é:

$\varepsilon_{a b c}=\left\{\begin{array}{cl}+1 & \text { se }\{a, b, c\}=\{1,2,3\},\{2,3,1\} \text { ou }\{3,1,2\} \\ -1 & \text { se }\{a, b, c\}=\{3,2,1\},\{1,3,2\} \text { ou }\{2,1,3\} \\ 0 & \text { se } a=b, b=c, \text { ou } c=a\end{array}\right.$

Uma observação importante que merece ser feita aqui é a seguinte: o grupo $\mathrm{SU}(2)$ possui 3 geradores, as

\footnotetext{
1 Dadas duas matrizes $A$ e $B$ de mesma ordem, a relação de anticomutação entre elas é dada por $\{A, B\}=A B+B A$.
} 
matrizes de Pauli, mas as matrizes do grupo são de ordem 2. Será se existe uma representação do grupo $\mathrm{SU}(2)$, mas de modo que as matrizes sejam de ordem 3 ? Veja então que, se definirmos três matrizes $\Sigma_{1}, \Sigma_{2}$ e $\Sigma_{3}$ da seguinte maneira

$$
\begin{aligned}
\Sigma_{1} & =\left(\begin{array}{ccc}
0 & 0 & 0 \\
0 & 0 & i \\
0 & -i & 0
\end{array}\right), \\
\Sigma_{2} & =\left(\begin{array}{ccc}
0 & 0 & -i \\
0 & 0 & 0 \\
i & 0 & 0
\end{array}\right), \\
\Sigma_{3} & =\left(\begin{array}{ccc}
0 & i & 0 \\
-i & 0 & 0 \\
0 & 0 & 0
\end{array}\right),
\end{aligned}
$$

as seguinte relações de comutação são válidas

$$
\begin{aligned}
& {\left[\Sigma_{1}, \Sigma_{2}\right]=\Sigma_{1} \Sigma_{2}-\Sigma_{2} \Sigma_{1}=i \Sigma_{3},} \\
& {\left[\Sigma_{2}, \Sigma_{3}\right]=\Sigma_{2} \Sigma_{3}-\Sigma_{3} \Sigma_{2}=i \Sigma_{1},} \\
& {\left[\Sigma_{3}, \Sigma_{1}\right]=\Sigma_{3} \Sigma_{1}-\Sigma_{1} \Sigma_{3}=i \Sigma_{2} .}
\end{aligned}
$$

Note ainda que todas as matrizes $\Sigma$ são hermitianas e possuem traço nulo, assim como as matrizes de Pauli. De maneira simplificada podemos, portanto, escrever a seguinte relação para as matrizes $\Sigma$,

$$
\left[\Sigma_{a}, \Sigma_{b}\right]=\Sigma_{a} \Sigma_{b}-\Sigma_{b} \Sigma_{a}=i \sum_{c=1}^{3} \varepsilon_{a b c} \Sigma_{c}
$$

com $a, b, c \in\{1,2,3\}$. Estamos diante, desta forma, de uma representação do grupo $\mathrm{SU}(2)$ com matrizes de ordem 3. Esta representação, como já vimos, é chamada de representação adjunta.

Em física de interações fundamentais a ideia de representação adjunta tem um papel fundamental, pois veja que, a interação forte possui simetria regida pelo grupo SU(3), que possui 8 geradores (como veremos mais adiante), e em sua representação adjunta as matrizes do grupo são matrizes quadradas de ordem 8. A interação forte possui 8 gluons como partículas mediadoras e, estando o grupo na representação adjunta, cada gerador estará relacionado a uma partícula mediadora da interação. Analogamente, a interação fraca possui simetria regida pelo grupo $\mathrm{SU}(2)$, que na representação adjunta possui 3 matrizes de ordem 3 como seus geradores, cada um destes geradores estando relacionados aos bósons vetoriais $W^{+}, W^{-}$e $Z^{0}$, que são as partículas mediadoras da interação fraca.

Veja também que calculando as seguintes quantidades

$$
\begin{aligned}
& \frac{\vec{\sigma}^{2}}{2}=\sum_{i=1}^{3} \frac{\sigma_{i}}{2} \cdot \frac{\sigma_{i}}{2}=\left(\frac{\sigma_{1}}{2}\right)^{2}+\left(\frac{\sigma_{2}}{2}\right)^{2}+\left(\frac{\sigma_{3}}{2}\right)^{2}=\frac{3}{4} I_{2} \\
& \vec{\Sigma}^{2}=\sum_{i=1}^{3} \Sigma_{i} \cdot \Sigma_{i}=\Sigma_{1}^{2}+\Sigma_{2}^{2}+\Sigma_{3}^{2}=2 I_{3}
\end{aligned}
$$

podemos identificar os fatores que acompanham a matriz identidade como $\frac{3}{4}=\frac{1}{2}\left(\frac{1}{2}+1\right)$, bem como $2=1(1+1)$, que, de modo geral, pode ser escrito como, $s(s+1)$. O leitor que já estudou mecânica quântica, consegue então perceber claramente que a álgebra su(2) descreve o spin, em mecânica quântica, sendo a quantidade $j(j+1)$ justamente o autovalor do operador momento angular $\vec{J}^{2}$.

O papel da mecânica quântica consiste justamente em adicionar o fator $\hbar$ às matrizes $\frac{\sigma_{i}}{2}$ a fim de produzir o operador de spin $S_{i}=\hbar\left(\frac{\sigma_{i}}{2}\right)$, que obedece à seguinte relação de comutação $\left[S_{i}, S_{j}\right]=i \epsilon_{i j k} S_{k}$, tal qual a relação 116 .

\section{Grupo $\mathrm{SO}(3)$ e as Rotações em Três Dimenões}

Nesta seção estudaremos o caso do grupo $\mathrm{SO}(3)$, que é basicamente uma extensão do grupo de rotações em duas dimensões (o grupo $\mathrm{SO}(2)$ ) para três dimensões. Apesar de estarmos lidando também com um grupo composto somente por matrizes ortogonais com determinante igual à unidade, algumas propriedades especiais desse grupo, que emergem por conta da dimensionalidade das matrizes, devem ser ressaltadas.

Primeiramente é importante ressaltar que o grupo $\mathrm{SO}(3)$ reúne todas as rotações realizadas no espaço euclidiano tridimensional real e que estas estão associadas a 3 tipos de geradores. Cada gerador está, portanto, relacionado à rotação em torno de cada um dos eixos perpendiculares aos planos cartesianos.

As matrizes responsáveis pela rotação de um dado vetor em torno de cada um dos eixos do $\mathbb{R}^{3}$ são as seguintes:

$$
\begin{aligned}
& R_{z}(\theta)=\left(\begin{array}{ccc}
\cos \theta & \operatorname{sen} \theta & 0 \\
-\operatorname{sen} \theta & \cos \theta & 0 \\
0 & 0 & 1
\end{array}\right), \\
& R_{y}(\beta)=\left(\begin{array}{ccc}
\cos \beta & 0 & -\operatorname{sen} \beta \\
0 & 1 & 0 \\
\operatorname{sen} \beta & 0 & \cos \beta
\end{array}\right), \\
& R_{x}(\alpha)=\left(\begin{array}{ccc}
1 & 0 & 0 \\
0 & \cos \alpha & \operatorname{sen} \alpha \\
0 & -\operatorname{sen} \alpha & \cos \alpha
\end{array}\right) .
\end{aligned}
$$

Estas matrizes são as conhecidas rotações de Euler. Para mais detalhes acerca da construção de tais matrizes, veja o apêndice. Desta forma, o elemento geral do grupo $\mathrm{SO}(3)$ é dado pela multiplicação das matrizes de Euler, isto é, $R(\alpha, \beta, \theta)=R_{z}(\alpha) R_{y}(\beta) R_{z}(\theta)$. Uma verificação direta simples pode mostrar que o grupo $\mathrm{SO}(3)$, diferentemente do grupo $\mathrm{SO}(2)$, não é abeliano, como podemos ver também na Figura (6).

Em segundo lugar, uma vez que as matrizes de $\mathrm{SO}(3)$ possuem todas determinante igual à unidade, 

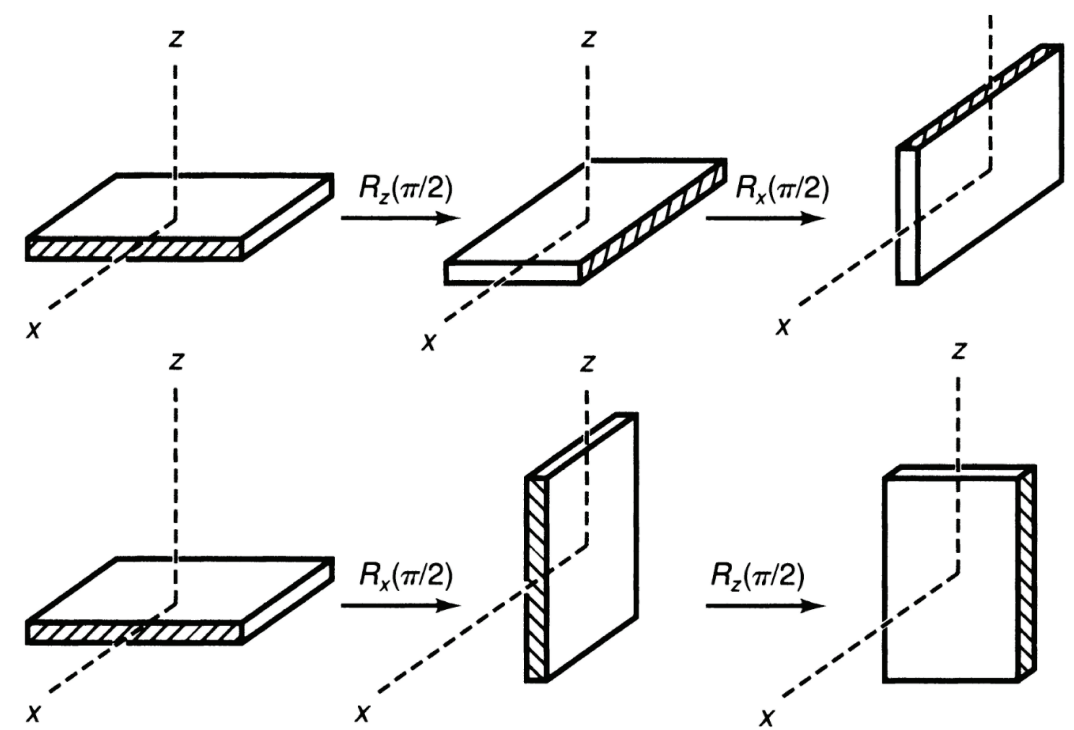

Figura 6: Não comutatividade das rotações em três dimensões (Fonte [14]).

sabemos que existe uma matriz $\Omega$ de traço nulo cuja exponencial gerará todos os elementos de $\mathrm{SO}(3)$. Usando um procedimento análogo ao que foi desenvolvido para o grupo $\mathrm{SO}(2)$, chegamos a condição 28 , que define agora uma matriz de ordem 3 antissimétrica. Esta matriz $\Omega$ pode ser expressa como:

$$
\Omega=a \Lambda_{1}+b \Lambda_{2}+c \Lambda_{3},
$$

sendo $a, b, c \in \mathbb{R}$ e as matrizes $\Lambda_{1}, \Lambda_{2}$ e $\Lambda_{3}$ definidas da seguinte forma:

$$
\begin{aligned}
\Lambda_{3} & =\left(\begin{array}{ccc}
0 & 1 & 0 \\
-1 & 0 & 0 \\
0 & 0 & 0
\end{array}\right), \\
\Lambda_{2} & =\left(\begin{array}{ccc}
0 & 0 & -1 \\
0 & 0 & 0 \\
1 & 0 & 0
\end{array}\right), \\
\Lambda_{1} & =\left(\begin{array}{ccc}
0 & 0 & 0 \\
0 & 0 & 1 \\
0 & -1 & 0
\end{array}\right) .
\end{aligned}
$$

As matrizes $\Lambda_{1}, \Lambda_{2}$ e $\Lambda_{3}$ são as matrizes geradoras do grupo $\mathrm{SO}(3)$ e formam uma base para todas as matrizes antissimétricas em três dimensões. Tais matrizes podem ainda ser obtidas através da relação (36). Além disso note que as matrizes (121), 120 e 119 podem ser obtidas através da exponenciação dos geradores. Veja agora que, se calcularmos as relações de comutação entre os geradores de $\mathrm{SO}(3)$, obteremos:

$$
\begin{aligned}
& {\left[\Lambda_{1}, \Lambda_{2}\right]=\Lambda_{1} \Lambda_{2}-\Lambda_{2} \Lambda_{1}=\Lambda_{3},} \\
& {\left[\Lambda_{2}, \Lambda_{3}\right]=\Lambda_{2} \Lambda_{3}-\Lambda_{3} \Lambda_{2}=\Lambda_{1},} \\
& {\left[\Lambda_{3}, \Lambda_{1}\right]=\Lambda_{3} \Lambda_{1}-\Lambda_{1} \Lambda_{3}=\Lambda_{2},}
\end{aligned}
$$

que, assim como foi feito no caso do grupo $\mathrm{SU}(2)$, pode ser generalizado para:

$$
\left[\Lambda_{a}, \Lambda_{b}\right]=\Lambda_{a} \Lambda_{b}-\Lambda_{b} \Lambda_{a}=\sum_{c=1}^{3} \epsilon_{a b c} \Lambda_{c}
$$

com $a, b, c \in\{1,2,3\}$. A álgebra expressa pelo grupo $\mathrm{SO}(3)$ é similar à álgebra exibida pelo grupo $\mathrm{SU}(2)$. Podemos ainda estabelecer a seguinte relação entre seus geradores

$$
\Lambda_{i} \longleftrightarrow-i \Sigma_{i}
$$

Neste momento somos tentados a inferir que há uma correspondência um a um entre os elementos de $\mathrm{SO}(3)$ e $\mathrm{SU}(2)$, o que categorizaria a existência de um isomorfismo entre os dois grupos, contudo tal inferência não é verdade. Se considerarmos, por exemplo, duas rotações, uma de $2 \pi$ e outra de $4 \pi$, na linguagem de $\mathrm{SO}(3)$ as matrizes que representam tais rotações são ambas matrizes identidade de ordem 3, enquanto que na linguagem de $\mathrm{SU}(2)$ teremos uma matriz identidade multiplicada por $(-1)$ e a própria identidade, respectivamente. Portanto a correspondência é de 2 para 1. Dizemos assim que os grupos $\mathrm{SO}(3)$ e $\mathrm{SU}(2)$ são localmente isomorfos.

\section{Quarks e o Grupo SU(3)}

Nesta seção estudaremos o grupo SU(3). Não nos deteremos a discorrer sobre os aspectos históricos que levaram à associação da simetria regida pelo grupo SU(3) com os quarks. O leitor interessado em uma discussão histórica e em aspectos fenomenológicos acerca do modelo de quarks pode consultar a referência [17.

Assim como o grupo $\mathrm{SO}(3)$, que é uma extensão do grupo $\mathrm{SO}(2)$ para três dimensões, o grupo $\mathrm{SU}(3)$ é uma extensão do grupo $\mathrm{SU}(2)$ para três dimensões. 
Como mostramos anteriormente para o grupo $\mathrm{SU}(2)$, a álgebra que rege o grupo das matrizes especiais unitárias é governada por matrizes hermitianas. No caso do grupo $\mathrm{SU}(3)$, a matriz $\Omega$ cuja exponenciação dará origem aos elementos do grupo SU(3) é tal que

$$
\Omega^{\dagger}=\Omega
$$

com $\Omega \in \mathbb{M}(3, \mathbb{C})$. O vínculo acima imposto sob a matriz $\Omega$ a simplifica à seguinte forma geral:

$$
\Omega=\left(\begin{array}{ccc}
a & b & c \\
b^{*} & e & f \\
c^{*} & f^{*} & i
\end{array}\right)
$$

$\mathrm{O}$ vínculo da hermiticidade de $\Omega$ impõe que $\{a, e, i\} \in$ $\mathbb{R}$ enquanto que $\{b, c, f\} \in \mathbb{C}$. Dessa forma, podemos expressar a matriz $\Omega$, de maneira mais geral, como:

$$
\Omega=\left(\begin{array}{ccc}
a & \beta_{1}-i \beta_{2} & \gamma_{1}-i \gamma_{2} \\
\beta_{1}+i \beta_{2} & e & \phi_{1}-i \phi_{2} \\
\gamma_{1}+i \gamma_{2} & \phi_{1}+i \phi_{2} & i
\end{array}\right)
$$

Existe certa arbitrariedade na forma como construir a matriz $\Omega$. Aqui, escolhemos a construção mais utilizada em física de partículas, que consiste em definir $b=\beta_{1}-$ $i \beta_{2}, c=\gamma_{1}-i \gamma_{2}$, bem como $f=\phi_{1}-i \phi_{2}$. Podemos então escrever a matriz $\Omega$ como uma soma de seus geradores $\lambda_{i}$, da seguinte maneira:

$\Omega=\beta_{1} \lambda_{1}+\beta_{2} \lambda_{2}+a \lambda_{3}+\gamma_{1} \lambda_{4}+\gamma_{2} \lambda_{5}+\phi_{1} \lambda_{6}+\phi_{2} \lambda_{7}+e \lambda_{8}$

com as matrizes $\lambda_{i}$ definidas como

$$
\begin{gathered}
\lambda_{1}=\left(\begin{array}{lll}
0 & 1 & 0 \\
1 & 0 & 0 \\
0 & 0 & 0
\end{array}\right), \lambda_{2}=\left(\begin{array}{ccc}
0 & -i & 0 \\
i & 0 & 0 \\
0 & 0 & 0
\end{array}\right), \\
\lambda_{3}=\left(\begin{array}{ccc}
1 & 0 & 0 \\
0 & -1 & 0 \\
0 & 0 & 0
\end{array}\right), \lambda_{4}=\left(\begin{array}{lll}
0 & 0 & 1 \\
0 & 0 & 0 \\
1 & 0 & 0
\end{array}\right), \\
\lambda_{5}=\left(\begin{array}{ccc}
0 & 0 & -i \\
0 & 0 & 0 \\
i & 0 & 0
\end{array}\right), \lambda_{6}=\left(\begin{array}{lll}
0 & 0 & 0 \\
0 & 0 & 1 \\
0 & 1 & 0
\end{array}\right), \\
\lambda_{7}=\left(\begin{array}{ccc}
0 & 0 & 0 \\
0 & 0 & -i \\
0 & i & 0
\end{array}\right), \lambda_{8}=\frac{1}{\sqrt{3}}\left(\begin{array}{ccc}
1 & 0 & 0 \\
0 & 1 & 0 \\
0 & 0 & -2
\end{array}\right) .
\end{gathered}
$$

O fator $1 / \sqrt{3}$ em $\lambda_{8}$ foi posto para manter a propriedade

$$
\operatorname{tr}\left(\lambda_{i} \lambda_{j}\right)=2 \delta_{i j}
$$

com $i, j=\{1,2, \ldots, 8\}$. Note que todas as matrizes $\lambda_{i}$ são hermitianas e tem traço nulo, assim como as matrizes de Pauli. A relação 139 estabelece um princípio de ortogonalidade em $\mathrm{SU}(3)$, além disso, um cálculo simples e direto nos mostra que a seguinte relação de comutação

$$
\left[\lambda_{i}, \lambda_{j}\right]=\sum_{k=1}^{3} 2 i \epsilon_{i j k} \lambda_{k}
$$

é válida para o grupo $\mathrm{SU}(3)$. No entanto, se não nos restringirmos somente às matrizes $\lambda_{1}, \lambda_{2}$ e $\lambda_{3}$ a relação de comutação ganha uma nova forma e passa a ser escrita como:

$$
\left[\lambda_{i}, \lambda_{j}\right]=\sum_{k=1}^{8} 2 i f_{i j k} \lambda_{k}
$$

O parâmetro $f_{i j k}$ é chamado de constante de estrutura do grupo $\mathrm{SU}(3)$, bem como o tensor de Levi-Civita $\epsilon_{i j k}$ é a constante de estrutura dos grupos $\mathrm{SU}(2)$ e $\mathrm{SO}(3)$. Analogamente, um cálculo direto mostra que a estrutura de anticomutador inerente às matrizes $\lambda$ pode ser expressa através da relação:

$$
\left\{\lambda_{i}, \lambda_{j}\right\}=\frac{4}{3} \delta_{i j} I+\sum_{k=1}^{8} 2 d_{i j k} \lambda_{k},
$$

sendo os coeficientes $d_{i j k}$ simétricos com relação a permutação dos índices, isto é,

$$
d_{i j k}=d_{i k j}=d_{j i k}=\ldots,
$$

enquanto que os coeficientes $f_{i j k}$ são antissimétricos perante a permutação de quaisquer dois índices, ou seja,

$$
f_{i j k}=-f_{j i k}=-f_{i k j} \text {. }
$$

Os valores não nulos da constante de estrutura $f_{i j k}$ bem como da constante $d_{i j k}$ estão listados na tabela abaixo.

\begin{tabular}{cccc}
\hline$i j k$ & $f_{i j k}$ & $i j k$ & $d_{i j k}$ \\
\hline 123 & 1 & 118 & $1 / \sqrt{3}$ \\
147 & $1 / 2$ & 146 & $1 / 2$ \\
156 & $-1 / 2$ & 157 & $1 / 2$ \\
246 & $1 / 2$ & 228 & $1 / \sqrt{3}$ \\
257 & $1 / 2$ & 247 & $-1 / 2$ \\
345 & $1 / 2$ & 256 & $1 / 2$ \\
367 & $-1 / 2$ & 338 & $1 / \sqrt{3}$ \\
458 & $\sqrt{3} / 2$ & 344 & $1 / 2$ \\
678 & $\sqrt{3} / 2$ & 355 & $1 / 2$ \\
& & 366 & $-1 / 2$ \\
& & 377 & $-1 / 2$ \\
& & 448 & $-1 /(2 \sqrt{3})$ \\
& & 558 & $-1 /(2 \sqrt{3})$ \\
& & 668 & $-1 /(2 \sqrt{3})$ \\
& & 778 & $-1 /(2 \sqrt{3})$ \\
& & 888 & $-1 / \sqrt{3}$ \\
\hline
\end{tabular}

\section{Oscilador Harmônico N-dimensional e o Grupo SU(N)}

Agora que já galgamos um bom caminho acerca de alguns dos grupos mais fundamentais por trás da descrição de sistemas físicos, voltemos ao exemplo inicial 
que demos na seção 2 deste trabalho. Como já foi dito e mostrado, o oscilador harmônico descrito em (1) possui simetria $\mathrm{O}(\mathrm{N})$, isto é, se definirmos

$$
p=\left(\begin{array}{c}
p_{1} \\
\cdot \\
\cdot \\
\cdot \\
p_{n}
\end{array}\right) \text { e } \quad x=\left(\begin{array}{c}
x_{1} \\
\cdot \\
\cdot \\
\cdot \\
x_{n}
\end{array}\right) \text {, }
$$

uma transformação $p^{\prime}=R p$ e e $x^{\prime}=R x$ deixará a energia do oscilador harmônico invariante se $R \in \mathrm{O}(\mathrm{N})$. Vamos trabalhar com mais detalhes agora alguns casos particulares.

\subsection{Oscilador harmônico unidimensional}

O oscilador harmônico unidimensional é um sistema trabalhado tradicionalmente em qualquer livro texto de mecânica clássica e mecânica quântica. Tal sistema será aqui utilizado como base para os sistemas subsequentes. Considere então o hamiltoniano que descreve um oscilador harmônico unidimensional, dado por:

$$
H=\frac{p^{2}}{2 m}+\frac{1}{2} k x^{2} .
$$

Vamos então definir as seguinte quantidades adimensionais:

$$
P=\frac{p}{\sqrt{2 m E_{0}}} \quad e \quad X=\sqrt{\frac{k}{2 E_{0}}} x,
$$

sendo $E_{0}$ a energia do oscilador. Substituindo as novas variáveis 147) no hamiltoniano do oscilador 146, obtemos

$$
H=E_{0}\left(P^{2}+X^{2}\right)
$$

Podemos ainda definir uma nova variável $\Pi$ da seguinte maneira:

$$
\Pi=P-i X
$$

de modo que, sabendo que $P$ e $X$ são quantidades inteiramente reais, $\Pi^{*}=P-i X$. Sendo assim, o produto

$$
\begin{aligned}
\Pi^{*} \Pi & =(P+i X)(P-i X) \\
& =P^{2}+X^{2}+i[X, P],
\end{aligned}
$$

nos permite reescrever o hamiltoniano do sistema como

$$
H=E_{0}\left(\Pi^{*} \Pi-i[X, P]\right) .
$$

Aqui reside a grande diferença entre a mecânica newtoniana e a mecânica quântica no que diz respeito ao estudo do oscilador harmônico. Na mecânica newtoniana o comutador $[X, P]=0$, enquanto que na mecânica quântica, fazendo uso da relação de comutação $[\hat{x}, \hat{p}]=$ $i \hbar \hat{I}$, o comutador $[\hat{X}, \hat{P}]=(i / 2) \hat{I}$, de modo que,

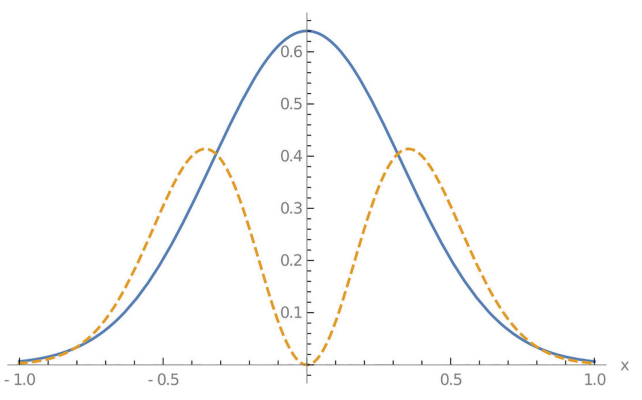

Figura 7: Densidade de probabilidade para o estado fundamental (linha contínua) e para o primeiro estado excitado (linha tracejada)

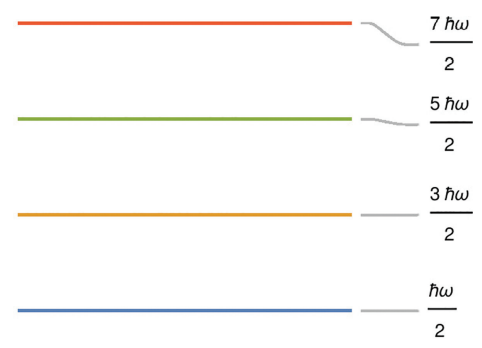

Figura 8: Os primeiros quatro níveis de energia para o oscilador harmônico quântico unidimensional.

para a mecânica quântica, o hamiltoniano do oscilador harmônico passa a ser escrito como

$$
\hat{H}=\hbar \omega\left(\hat{a}^{\dagger} \hat{a}+\frac{1}{2}\right) .
$$

Para a obtenção do hamiltoniano acima, consideramos $E_{0}=\hbar \omega$ e identificamos o operador de aniquilação $\hat{a}=\Pi$.

Para o caso quântico, lembremos que o operador número $\hat{N}=\hat{a}^{\dagger} \hat{a}$ é hermitiano, tendo portanto somente autovalores $n \in \mathbb{R}$ e $n \geq 0$. Desta forma, o valor de menor energia para o sistema é quando $n=0$ e portanto $E_{n=0}=(1 / 2) \hbar \omega$. É possível ainda mostrar que $n$ deve ser um número inteiro. Desta forma, o primeiro estado excitado do oscilador harmônico é quando $n=1$, logo, $E_{n=1}=(3 / 2) \hbar \omega$. Como $\hat{N}|n\rangle \geq 0$, avaliemos o caso da igualdade em zero. Como

$$
\hat{N}|0\rangle=\hat{a}^{\dagger} \hat{a}|0\rangle=0 \Rightarrow\left\langle 0\left|\hat{a}^{\dagger} \hat{a}\right| 0\right\rangle=0,
$$

temos que $\hat{a}|0\rangle=0$. Atuando $\langle x|$ pela esquerda, isto é,

$$
\langle x|\hat{a}| 0\rangle=\hat{a} \psi_{0}(x)=0,
$$

e substituindo $\hat{a}=P-i X$, chegamos à seguinte equação diferencial

$$
\frac{\hbar}{\sqrt{2 m \hbar \omega}} \frac{d \psi_{0}(x)}{d x}=-\sqrt{\frac{m \omega}{2 \hbar}} x \psi_{0}(x),
$$

cuja solução nos dá a função de onda do oscilador harmônico quântico unidimensional como sendo

$$
\psi_{0}(x)=\alpha e^{-\frac{m \omega}{2 \hbar} x^{2}}
$$


A constante $\alpha$ pode ser obtida através da condição de normalização da função de onda, de modo que $\alpha=(m \omega / \pi \hbar)^{1 / 4}$. Note que a função de onda exibe o comportamento de uma gaussiana centrada na origem. A função de onda para o primeiro estado excitado pode ser obtida através da aplicação do operador de criação $\hat{a}^{\dagger}$ na função de onda para o estado fundamental.

As equações 151 e 152 foram reescritas a partir de um hamiltoniano para o oscilador harmônico, mas note que tais equações expressam uma invariância por transformações do grupo unitário, isto é, se definirmos $\hat{a}^{\prime}=M \hat{a}$, o hamiltoniano 152 se manterá invariante se a matriz $M$ for unitária. Ou seja, o oscilador harmônico possui na verdade simetria perante transformações do grupo unitário, que é um grupo bem maior que o grupo das transformações ortogonais.

\subsection{Oscilador harmônico bidimensional isotrópico}

O oscilador harmônico bidimensional isotrópico é descrito pelo seguinte hamiltoniano:

$$
H=\frac{1}{2 m}\left(p_{x}^{2}+p_{y}^{2}\right)+\frac{1}{2} k\left(x^{2}+y^{2}\right) .
$$

A isotropia é expressa pelo fato de que a constante elástica $k$ é a mesma tanto para a componente $x$ do movimento quanto para a componente $y$. Aqui, procederemos exatamente como fizemos para o oscilador unidimensional. Vamos definir as seguintes quantidades adimensionais:

$$
\begin{aligned}
& P_{x}=\frac{p_{x}}{\sqrt{2 m E_{0}}}, \quad X=\sqrt{\frac{k}{2 E_{0}}} x \\
& P_{y}=\frac{p_{y}}{\sqrt{2 m E_{0}}}, \quad Y=\sqrt{\frac{k}{2 E_{0}}} y .
\end{aligned}
$$

Analogamente, definiremos as variáveis $\Pi_{x}$ e $\Pi_{y}$ como

$$
\begin{aligned}
& \Pi_{x}=P_{x}-i X \\
& \Pi_{y}=P_{y}-i Y .
\end{aligned}
$$

Desenvolvendo, portanto, o hamiltoniano em 157) incorporando as novas variáveis, chegamos à seguinte expressão

$$
H=E_{0}\left(\Pi_{x}^{*} \Pi_{x}+\Pi_{y}^{*} \Pi_{y}-i\left[X, P_{x}\right]-i\left[Y, P_{y}\right]\right) .
$$

Salientamos novamente aqui que a diferença entre a mecânica clássica e a mecânica quântica está no fato de que as relações de comutação entre coordenada e momento se anulam no regime clássico. Para o caso quântico vale ressaltar uma diferença pontual do caso bidimensional para com o caso unidimensional. Note que, fazendo uso das relações de comutação entre coordenada e momentum e tomando $E_{0}=\hbar \omega$ chegamos à seguinte expressão para o hamiltoniano:

$$
\hat{H}=\hbar \omega\left(\hat{a}_{x}^{\dagger} \hat{a}_{x}+\hat{a}_{y}^{\dagger} \hat{a}_{y}+1\right) .
$$

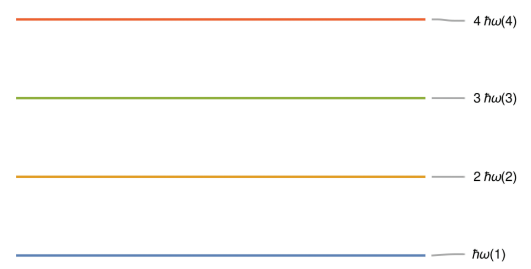

Figura 9: Níveis de energia e degenerescências (entre parêntesis) para o oscilador harmônico quântico bidimensional isotrópico.

Façamos agora algumas considerações a fim de apontar as diferenças entre os casos unidimensional e bidimensional. Primeiramente, é possível ver que o mínimo de energia para o caso bidimensional não é mais $E_{n=0}^{u n i}=$ $(1 / 2) \hbar \omega$, mas sim $E_{n=0}^{b i}=\hbar \omega$, uma diferença caracterizada por um fator multiplicativo de 2. Tal qual o caso unidimensional, vemos que no caso bidimensional o hamiltoniano de partida 157 é invariante por transformações de $\mathrm{O}(2)$, enquanto que o hamiltoniano 162 é invariante por transformações de U(2). Dizemos então que a simetria $\mathrm{SO}(2)$ do hamiltoniano é uma simetria "estática", uma vez que relaciona as coordenadas e momento independentemente, enquanto que a simetria $\mathrm{SU}(2)$ é dita simetria dinâmica, pois esta simetria "mistura" coordenadas e momentos em uma única simetria.

Outra diferença crucial entre o caso unidimensional e o caso bidimensional é o fato de que, com exceção do estado fundamental, todos os demais estados são degenerados, isto é, existem vários estados quânticos com a mesma energia. Para verificarmos tal fato tomemos os autovalores do hamiltoniano em (163), o que nos dá a seguinte expressão para a energia do sistema:

$$
E\left(n_{x}, n_{y}\right)=\hbar \omega\left(n_{x}+n_{y}+1\right) .
$$

Veja então que o estado fundamental ocorre quando tanto $n_{x}$ quando $n_{y}$ são iguais a zero simultaneamente, assim temos $E_{0,0}=\hbar \omega$. No entanto, para o primeiro estado excitado, podemos ter $n_{x}=0$ e $n_{y}=1$, bem como $n_{x}=1$ e $n_{y}=0$, de modo que teremos dois estados com energia $E_{0,1}=E_{1,0}=2 \hbar \omega$. Dizemos então que o primeiro estado excitado do oscilador harmônico quântico bidimensional isotrópico possui degenerescência igual a 2. Podemos proceder de maneira análoga para os estados excitados subsequentes.

O cálculo para obter a função de onda para o estado fundamental no caso bidimensional é semelhante ao que foi feito para o caso unidimensional, com o único adicional de termos que usar duas condições, a saber, $\hat{a}_{x} \psi_{0}(x, y)=0$ e $\hat{a}_{y} \psi_{0}(x, y)=0$. Podemos então, de maneira análoga, obter a função de onda $\psi_{0}(x, y)$ para oscilador harmônico quântico bidimensional como

$$
\psi_{0}(x, y)=\sqrt{\frac{m \omega}{\pi \hbar}} e^{-\frac{m \omega}{2 \hbar} x^{2}} e^{-\frac{m \omega}{2 \hbar} y^{2}} .
$$

A fim de explicitarmos as degenerescências nos estados excitados, calcularemos as duas funções de onda cujas 


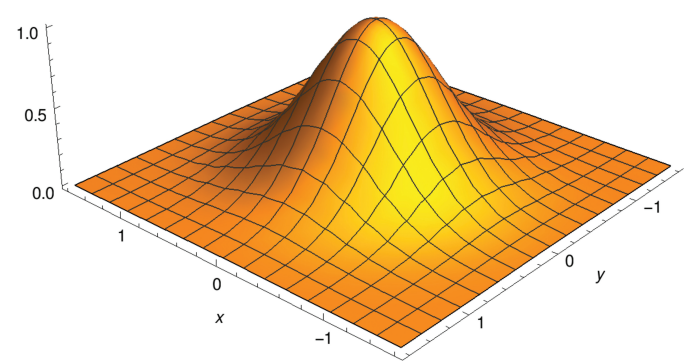

Figura 10: Densidade de probabilidade para o estado fundamental do oscilador harmônico quântico bidimensional isotrópico.

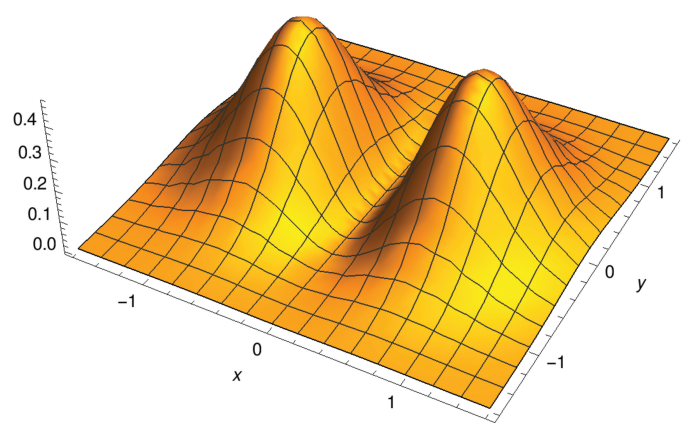

Figura 11: Densidade de probabilidade para o primeiro estado excitado $\left|\psi_{1}(x, y)\right|^{2}$.

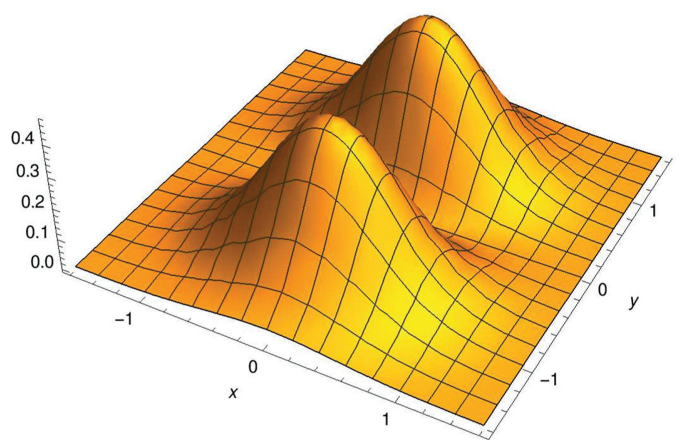

Figura 12: Densidade de probabilidade para o primeiro estado excitado $\left|\psi_{1}^{\prime}(x, y)\right|^{2}$.

energias são iguais a $\hbar \omega$. Para calcularmos estas funções de onda, aplicaremos os operadores de criação $\hat{a}_{x}^{\dagger}$ e $\hat{a}_{y}^{\dagger}$ na função de onda do estado fundamental. Desta forma, obtemos,

$$
\begin{aligned}
& \psi_{1}(x, y)=\frac{i \sqrt{2}}{\sqrt{\pi}} \frac{m \omega}{\hbar} x e^{-\frac{m \omega}{2 \hbar} x^{2}} e^{-\frac{m \omega}{2 \hbar} y^{2}} \\
& \psi_{1}^{\prime}(x, y)=\frac{i \sqrt{2}}{\sqrt{\pi}} \frac{m \omega}{\hbar} y e^{-\frac{m \omega}{2 \hbar} x^{2}} e^{-\frac{m \omega}{2 \hbar} y^{2}} .
\end{aligned}
$$

\subsection{Oscilador harmônico $\mathrm{N}$-dimensional isotrópico}

Uma extensão imediata do oscilador harmônico em duas dimensões permite que escrevamos o oscilador harmônico em N-dimensões como:

$$
H=\frac{1}{2 m} \sum_{i=1}^{N} p_{i} p_{i}+\frac{1}{2} k \sum_{i=1}^{N} x_{i} x_{i} .
$$

Efetuando as mesmas mudanças de variáveis realizadas no caso bidimensional, agora para um número $\mathrm{N}$ de dimensões obtemos, para o caso clássico

$$
H=E_{0}\left(\sum_{i=1}^{N} \Pi_{i}^{*} \Pi_{i}-i \sum_{i=1}^{N}\left[X_{i}, P_{i}\right]\right),
$$

enquanto para o caso quântico temos:

$$
\hat{H}=\hbar \omega\left(\sum_{i=1}^{N} \hat{a}_{i}^{\dagger} \hat{a}_{i}+\frac{N}{2}\right) .
$$

Vemos então que o hamiltoniano de partida do oscilador harmônico N-dimensional possui uma simetria explícita $\mathrm{O}(\mathrm{N})$, mas que apresenta uma simetria dinâmica mais geral, $\mathrm{U}(\mathrm{N})$, após uma mudança de variáveis.

\section{Conclusão}

Visando ser um possível material de apoio ao estudante de Graduação, seja de Bacharelado ou de Licenciatura, nesta contribuição buscamos abordar de maneira sucinta e intuitiva a poderosa ferramenta da teoria de grupos, focando nossa atenção em grupos que são de grande importância no estudo de física de partículas, e em geral de física de altas energias. Adotamos uma abordagem que parte de um grupo de Lie muito simples, comutativo, o grupo $\mathrm{SO}(2)$, relacionando este grupo com o princípio de invariância do produto escalar e estabelecendo a conexão deste com as rotações em duas dimensões. Nas seções subsequentes abordamos, os grupos $\mathrm{Sp}(2), \mathrm{SU}(2), \mathrm{SO}(3)$ e SU(3), sempre partindo de princípios de invariância e simetria para que assim um paralelo com a física, em especial com a física do modelo padrão, seja estabelecido. Discutimos ainda alguns aspectos importantes no que diz respeito ao oscilador harmônico isotrópico bidimensional e N-dimensional dentro da perspectiva da teoria de grupos.

Um dos pontos de grande importância no estudo de teoria de grupos consiste na fundamentação de um conhecimento mais sólido no que diz respeito à física básica. Outro ponto igualmente importante que é propiciado pelo estudo de teoria de grupos é a possibilidade de uma compreensão mais plena de tópicos de pesquisa em física, que muitas vezes estão fora do domínio de compreensão do estudante de graduação por requerer um arcabouço matemático muito sofisticado.

\section{Agradecimentos}

Gostariamos de agradecer aos revisores do artigo pelas valorosas sugestões e comentários. 


\section{Material Suplementar}

\section{Apêndice: Matrizes de Euler}

Neste apêndice mostraremos como são construídas as matrizes de Euler em uma abordagem algebrista, diferentemente da abordagem geométrica usualmente estudada. Para tal, considere o seguinte lema:

Lema: Seja $R \in \mathrm{SO}(\mathrm{n})$ com $n$ ímpar. Então, 1 é um auto valor de $R$ e, portanto, existe ao menos um vetor não nulo $v \in \mathbb{R}^{n}$ tal que $R v=v$.

Prova: Como $R \in \mathrm{SO}(\mathrm{n}), \operatorname{det}(R)=1$. Logo,

$$
\begin{aligned}
\operatorname{det}(I-R) & =\operatorname{det}\left[R\left(R^{T}-I\right)\right] \\
& =\operatorname{det}(R) \operatorname{det}\left[(R-I)^{T}\right] \\
& =\operatorname{det}(R-I) \\
& =\operatorname{det}[-(I-R)] \\
& =-\operatorname{det}(I-R),
\end{aligned}
$$

desta forma, $\operatorname{det}(I-R)=0$, que implica que 1 é autovalor de $R$, isto é, existe $v \in \mathbb{R}^{3}$ tal que $R v=v$.

De maneira mais simples isto quer dizer que, para todo grupo $\mathrm{SO}(\mathrm{n})$ com $n$ ímpar, existe um vetor $v \in \mathbb{R}^{n}$ cuja atuação de $R$ sob tal vetor o deixará invariante. No caso particular do grupo $\mathrm{SO}(3)$ existirá sempre um vetor que será deixado invariante perante atuação de uma matriz $R \in \mathrm{SO}(3)$. Ainda para o caso particular de $\mathrm{SO}(3)$, podemos dizer que o subespaço $V$ de $\mathbb{R}^{3}$ formado pelos vetores que são deixados invariantes perante atuação de $R \in \mathrm{SO}(3)(R \neq I)$ é unidimensional.

Veja ainda que o subespaço $V$ pode não ser o mesmo para matrizes $R$ distintas. Veja também que foi excluído o caso $R=I$, uma vez que qualquer vetor de $\mathbb{R}^{3}$ é invariante por $I$ e não apenas um subespaço unidimensional.

Vamos agora provar que o subespaço $V \subset \mathbb{R}^{3}$ formado pelos vetores que são deixados invariantes perante atuação de $R \in \mathrm{SO}(3)(R \neq I)$ é unidimensional. Para tal considere $R \neq I$ uma matriz qualquer de $\mathrm{SO}(3)$ e considere também

$$
V=\left\{v \in \mathbb{R}^{3} ; R v=v\right\} .
$$

Pelo lema acima demonstrado, sabemos que $V \neq \emptyset$ e que sua dimensão pode ser, portanto, 1, 2 ou 3. Note ainda que, se $v \in V$, então, aplicando $R^{T}$ à esquerda de $R v=v$, vemos que $R^{T} v=v$, uma vez que $R^{T} R=I$. Considere agora $V^{\perp}$ como sendo o conjunto de todos os vetores $u$ ortogonais à todos os vetores de $V$. Vamos ver agora que a aplicação de $R$ também deixa os vetores ortogonais à $v \in V$ invariantes, isto é, para todo $u \in V^{\perp}$ então $R u \in V^{\perp}$. Para tal, seja $v \in V$ e $u \in V^{\perp}$. Como $\vec{u} \cdot \vec{v}=0$, em notação matricial, temos

$$
0=U^{T} V=U^{T}\left(R^{T} V\right)=(R U)^{T} V
$$

Como isso vale para todo $v \in V$, temos que $R u \in V^{\perp}$. Por fim, vamos mostrar agora que a dimensão de $V$ tem que ser obrigatoriamente 1 , isto é, que os casos de $V$ com dimensão 2 e 3 não são possíveis.

Se a dimensão de $V$ fosse igual a $3, V$ seria idêntico ao $\mathbb{R}^{3}$ e neste caso $R v=v$ para todo $v \in \mathbb{R}^{3}$, o que implica em $R=I$, situação que foi excluída.

Considerando que $\operatorname{dim}(V)=2$, a dimensão de seu complemento ortogonal $V^{\perp}$ é igual a 1. Como mostramos que $V^{\perp}$ é invariante pela ação de $R \in \mathrm{SO}(3)$, então existe $\lambda \in \mathbb{R}$ tal que $R u=\lambda u$ para todo $u \in V^{\perp}$. A invariância de $u \in V^{\perp}$ por $R$ requer que $U^{T} U=$ $(R U)^{T}(R U)$. Usando a equação de autovalor chegamos a $U^{T} U=\lambda^{2} U^{T} U$, que nos dá que $\lambda= \pm 1$. Se $\lambda=+1$ então $u \in V$ ao invés de $u \in V^{\perp}$. Logo $\lambda=-1$. Neste caso a matriz $R$ teria dois autovalores iguais a +1 e um autovalor igual a -1 , o que a faria ter determinante igual a -1 , o que é uma contradição uma vez que $R \in \mathrm{SO}(3)$. Portanto a dimensão de $V$ só pode ser igual a 1 , como queríamos demonstrar.

Suponha agora que escolhemos em $\mathbb{R}^{3}$ uma base ortonormal formada pelos vetores $v, u_{1}$ e $u_{2} \operatorname{com} v \in V$ e $u_{1}, u_{2} \in V^{\perp}$. Como $v$ é deixado invariante pela ação de $R$, bem como $V^{\perp}$, então a matriz tem que ter a seguinte forma:

$$
R=\left(\begin{array}{lll}
1 & 0 & 0 \\
0 & ? & ? \\
0 & ? & ?
\end{array}\right)
$$

A submatriz $r$ formada pelos elementos indeterminados deve ser tal que, para todo $u \in V^{\perp}$ então $U^{T} U=$ $(r U)^{T}(r U)$, uma vez que a propriedade geral de $R$ é manter o produto escalar invariante. Logo $r$ deve ser ortogonal. Além disso, $1=\operatorname{det}(R)=\operatorname{det}(r)$. Sendo assim $r \in \mathrm{SO}(2)$ e tem a forma $19 p$. Portanto $R$ tem a forma apresentada em 121.

\section{Referências}

[1] P.A.M. Dirac, Proc. Roy. Soc. Lond. A 117, 610 (1928).

[2] J.J. Thomson, Phil. Mag. 44, 269 (1897).

[3] D. Griffiths, Introduction of Elementary Particles (Wiley-vch, Oregon, 2012), $2^{\mathrm{a}}$ ed.

[4] E. Rutherford, Lond. Edinb. Dubl. Phil. Mag. 37, 222 (1919).

[5] A. Einstein, Annalen der Physik 17, 6 (1905).

[6] A. Compton, Phys. Rev. 21, 5 (1923).

[7] P.A.M. Dirac, Proc. Roy. Soc. Lond. A 126, 360 (1930).

[8] H. Weyl, The theory of groups and quantum mechanics (Dover, New York, 1950).

[9] P.A.M. Dirac, Proc. Roy. Soc. Lond. A 133, 60 (1931).

[10] C.D. Anderson, Phys. Rev. 43, 6 (1933).

[11] M. Gell-Mann, Phys. Rev. 125, 3 (1962).

[12] L.S. Martin, Grupos de lie (Unicamp, Campinas, 2017).

[13] F.U. Coelho, Introdução à álgebra linear (Edusp, São Paulo, 2003), $3^{\mathrm{a}}$ ed. 
[14] J.J. Sakurai e J. Napolitano, Mecânica quântica moderna (Bookman, Porto Alegre 2012), $2^{a}$ ed.

[15] A.B. Jambersi e S. Silva, Rev. Bras. Ensino de Fís. 38, e2313 (2016).

[16] J. Vaz Jr. e R. Rocha Jr., Álgebras de Clifford e Espinores (Livraria da Física, São Paulo, 2012).

[17] C.O. Silva e P.L. Natti, Rev. Bras. Ensino de Fís. 29, $175,2007$. 